\title{
Seychelles: First Review Under the Extended Arrangement, Request for Modification of Performance Criteria, and Financing Assurances Review-Staff Report; Staff Supplement; and Press Release
}

In the context of the First Review Under the Extended Arrangement, request for Modification of Performance Criteria, and Financing Assurances Review with Seychelles, the following documents have been released and are included in this package:

- $\quad$ The staff report for the First Review Under the Extended Arrangement, Request for Modification of Performance Criteria, and Financing Assurances Review, prepared by a staff team of the IMF, following discussions that ended on May 17, 2010, with the officials of Seychelles on economic developments and policies. Based on information available at the time of these discussions, the staff report was completed on June 15, 2010. The views expressed in the staff report are those of the staff team and do not necessarily reflect the views of the Executive Board of the IMF.

- $\quad$ A Press Release summarizing the views of the Executive Board.

The documents listed below have been or will be separately released.

Letter of Intent sent to the IMF by the authorities of Seychelles*

Supplementary Memorandum of Economic, Financial and Structural Reform Policies by the authorities of Seychelles *

Technical Memorandum of Understanding*

*Also included in Staff Report

The policy of publication of staff reports and other documents allows for the deletion of market-sensitive information.

\author{
Copies of this report are available to the public from \\ International Monetary Fund • Publication Services \\ $70019^{\text {th }}$ Street, N.W. • Washington, D.C. 20431 \\ Telephone: (202) 623-7430 • Telefax: (202) 623-7201 \\ E-mail: publications@imf.org • Internet: http://www.imf.org
}

\section{International Monetary Fund \\ Washington, D.C.}




\title{
INTERNATIONAL MONETARY FUND
}

\section{SEYCHELLES}

\section{First Review Under the Extended Arrangement, Request for Modification of Performance Criteria, and Financing Assurances Review}

Prepared by the African Department

(In consultation with other Departments)

Approved by Roger Nord and Aasim Husain

June 15,2010

\begin{abstract}
A three-year arrangement under the Extended Fund Facility (EFF) for 225 percent of quota (SDR 19.8 million) was approved by the Executive Board on December 18, 2009.

SDR 3.08 million (35 percent of quota) has been disbursed, and SDR 2.2 million (25 percent of quota) is available upon completion of the first EFF review.
\end{abstract}

The program is on track. All quantitative performance criteria (PCs) at end-March 2010 were met and the two structural benchmarks at end-March and end-April 2010 were met.

Policy discussions were held in Victoria May 4-17, 2010. Staff met with President James Michel, Minister of Finance Danny Faure, Governor Pierre Laporte of the Central Bank of Seychelles (CBS), other senior government officials, and representatives of parliament, the private sector, civil society, and the diplomatic community. The mission comprised Jean Le Dem (head), Marie-Therese Trasino, Nikoloz Gigineishvili (all AFR), and Nkunde Mwase (SPR).

In the attached letter from President Michel and accompanying Supplementary Memorandum of Economic and Financial Policies (SMEFP) for 2010-12, the authorities request completion of the first program review under the EFF. The program has been updated in part to reflect better-than-expected government revenue outcome in the first quarter of 2010. New fiscal targets lock in half of projected additional revenue, while allowing additional spending in priority areas. The authorities are requesting modification of the quantitative performance criteria on the fiscal primary balance.

The authorities have implemented most of the recommendation of the 2008 safeguards assessment. The published 2009 CBS financial statements were prepared and audited in accordance with international standards. The update safeguards assessment, which is substantially complete, has found an improved safeguards framework at the CBS. Some vulnerabilities remain, however, that the CBS plans to address expeditiously. 


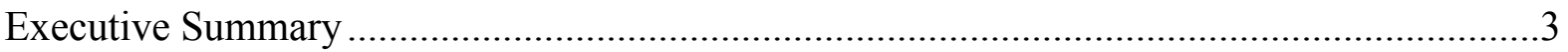

I. Recent Developments and Performance Under the Program ..................................................4

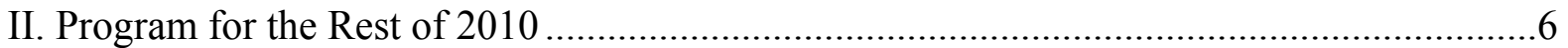

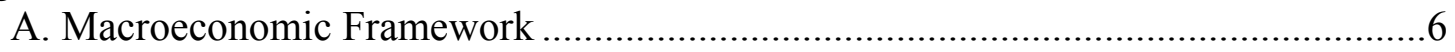

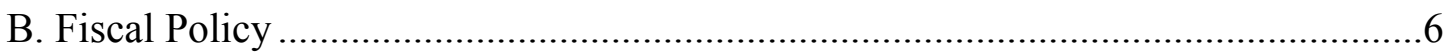

C. Monetary, Exchange Rate, and Financial Sector Policies.........................................

D. Public Enterprise Sector Reforms .........................................................................9

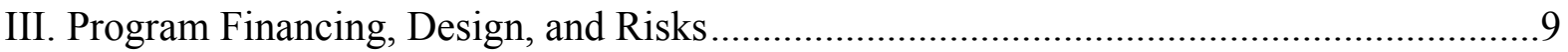

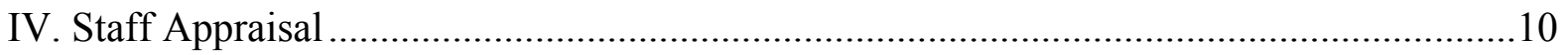

Tables

1. Selected Economic and Financial Indicators, 2007-12 ….............................................12

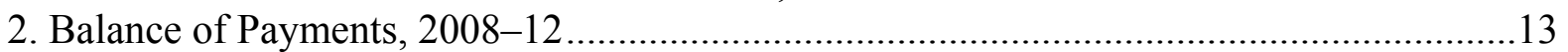

3. Consolidated Government Operations, 2008-12 ….....................................................

4. Monetary Survey and Central Bank Accounts, 2008-10.................................................16

5. Financial Soundness Indicators for the Banking Sector, 2007-10 …................................17

6. Fund Disbursements and Timing of Reviews Under the Proposed Three-Year EFF,

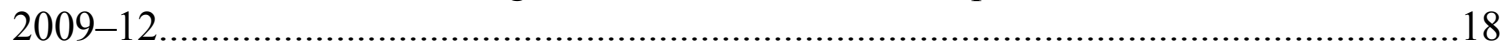

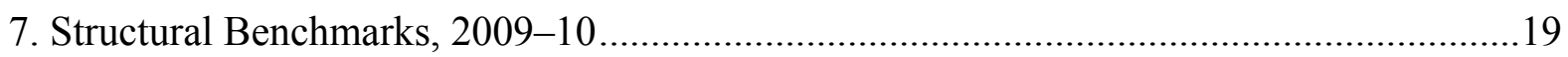

Figures

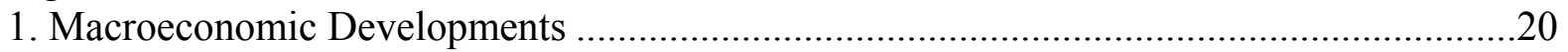

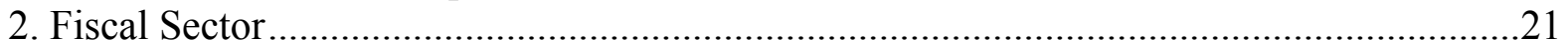

Appendices

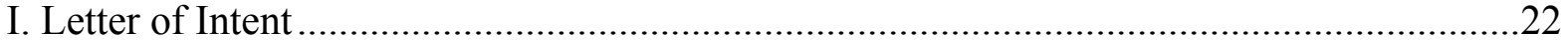

Attachment I. Supplementary Memorandum of Economic, Financial and Structural

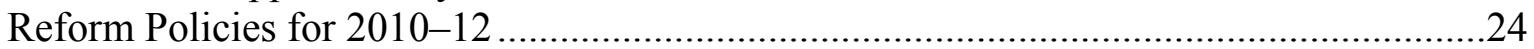

Attachment II. Technical Memorandum of Understanding ...................................................42 


\section{EXECUTIVE SUMMARY}

Macroeconomic performance under the program has been good. The Seychelles economy has proved remarkably resilient to the recent global crisis. Real GDP grew by 0.7 percent in 2009 and is projected to recover further to 4 percent in 2010 . Low inflation, a stable exchange rate and a steady rise in reserves, have boosted domestic confidence, further entrenching hard-won macroeconomic stabilization gains. All quantitative performance criteria at end-March 2010 were met.

The fiscal policy stance continues to be appropriate, facilitating a reduction in public debt. Revenue projections for 2010 were revised upwards by SR 124 million or 1.1 percent of GDP, and the primary balance target for 2010 was raised by SR 62 million to 7.4 percent of GDP, saving half the projected additional revenue. With the phased release of the contingency, additional capital expenditure is being directed towards much-needed infrastructural upgrading. Tax reforms are progressing: revised business tax legislation was enacted in January 2010, reducing rates and broadening the tax base; the sales tax base was expanded, cascading effects were reduced and concessional rates are being phased out. The introduction of a withholding-based personal income tax and VAT are on track for, respectively, July 2010 and January 2012.

Monetary policy is suitably designed to maintain inflation in low single digits. Within its operational framework of reserve money targeting, the central bank will continue to rely on repurchase agreements and deposit and credit auctions to guide short-term interest rates. However, the monetary transmission mechanism remains challenged by banks' excess reserves. Changes to the T-bill auction calendar are planned to enhance banks' ability to manage liquidity and strengthen the interbank market.

Structural reforms have progressed well, and program benchmarks were implemented as scheduled. Although quasi-fiscal risks stemming from public enterprises are currently contained, the government has commissioned a study of the optimal tariff policy, subjected the public utility to an independent operational audit, and engaged an international consulting firm to carry out a strategic assessment of the national airline. Efforts to enhance transparency and governance in state-owned entities are ongoing and their financial audits are subject to parliamentary oversight.

Good progress has been achieved in external public debt restructuring. The private debt exchange offer was successfully closed in February 2010, and the authorities are continuing good faith negotiations with the remaining non-Paris Club bilateral and other creditors. The program is financed for 2010 .

In light of strong performance through end-April 2010, compliance with the performance criteria, consistency with the Fund's Lending into Arrears policy, and satisfactory progress with the update safeguards assessment, staff recommends completion of the first EFF arrangement program and financing assurances reviews. 


\section{Recent Developments and Performance Under the Program}

1. Seychelles has weathered both the $\mathbf{2 0 0 8}$ debt crisis and the $\mathbf{2 0 0 9}$ global crisis. Aggressive discounting in the tourism industry, accounting for a quarter of economic activity, helped real GDP growth recover to 0.7 percent in 2009, a significant turnaround from the 7.6 percent contraction expected at the onset of the program. The current account deficit narrowed by a third to reach 35 percent of GDP in 2009. Imports contracted almost twice as fast as exports because of reduced domestic demand and a sharp decline in foreign direct investment (FDI) inflows from their 2008 peak.

\section{Macroeconomic stabilization appears to be taking hold on the back of}

rebounding domestic confidence. Higher visitor arrivals and a longer average stay through April 2010, by 13 percent and 5 percent respectively, point to a further recovery in activity. The rupee recovered strongly in 2009 from its overshooting following the 2008 float. Since October 2009, the nominal effective exchange rate has roughly stabilized, the depreciation against the US dollar being offset by the appreciation against the euro and the British pound. After the exchange rate pass-through triggered a rapid disinflation in 2009 , monthly inflation gradually returned to positive territory, although in May 2010 consumer prices were 3.6 percent below their level a year ago. Gross reserves increased to USD 183 million, or 1.9 months of prospective imports in May 2010.

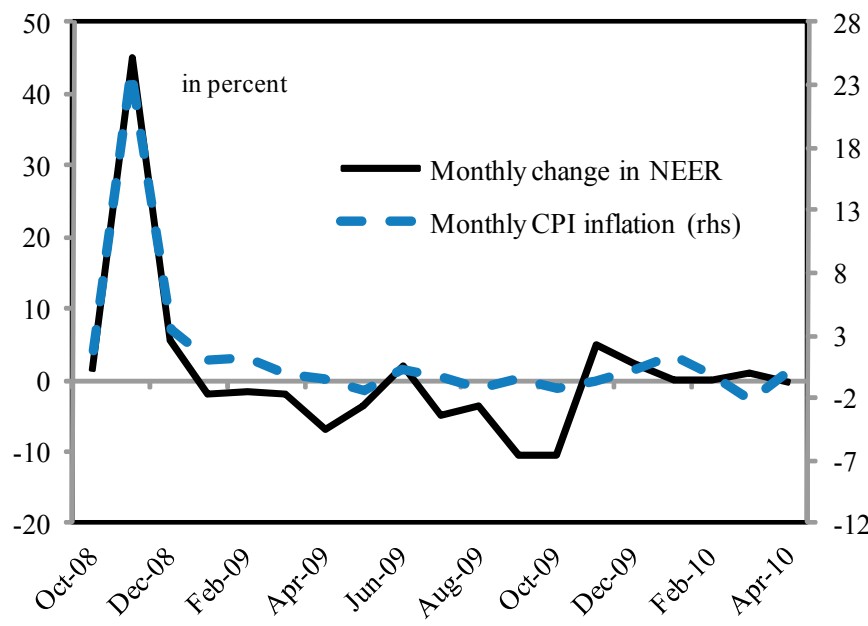

Source: Seychelles authorities and Fund staff estimates. 
Following strong appreciation in 2009, the exchange rate has depreciated somewhat.

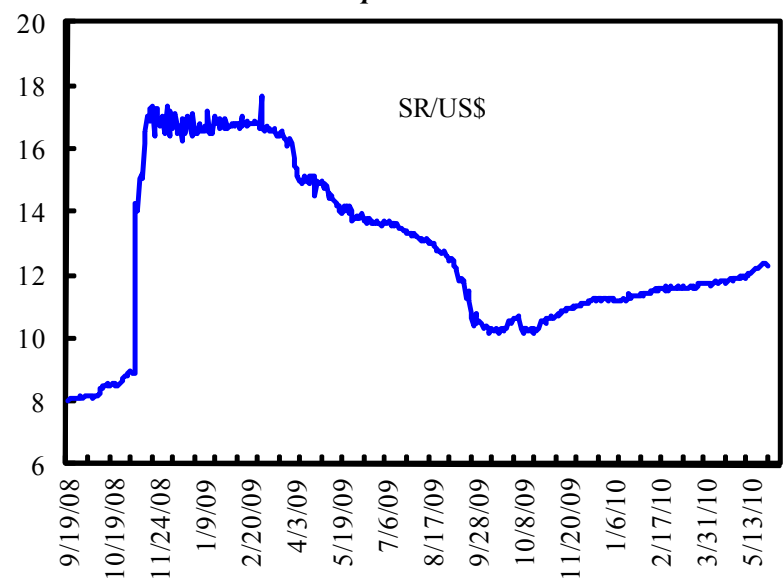

Source: Seychelles authorities and Fund staff estimates
Spreads between buying and seling rates have been on a downward trend as markets stabilized.

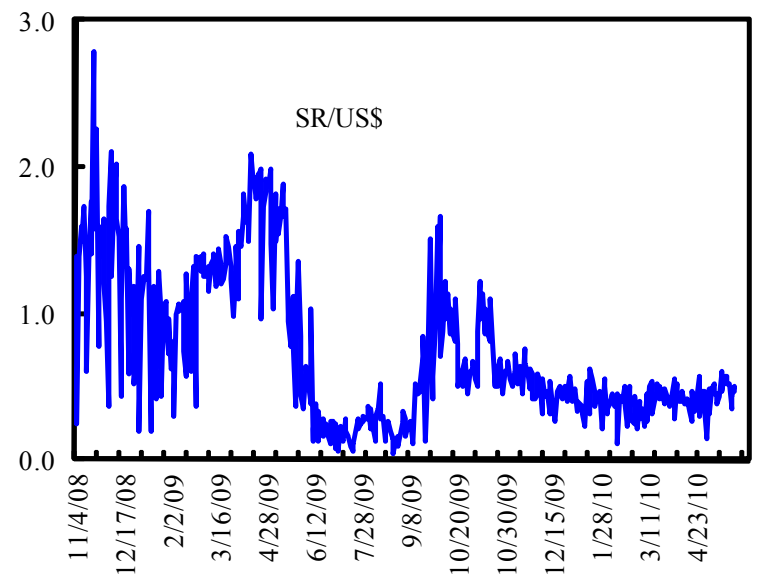

3. The fiscal policy stance is in line with the targeted public debt reduction. The primary surplus for the first quarter of 2010 exceeded the program target by 0.3 percentage points of GDP. Business tax receipts were driven by stronger-than-expected growth in 2009 while the rupee's depreciation against the dollar boosted trade and sales taxes on imports. On the expenditure side, delays in implementing the new wage grid and scheme of service for health and education and stricter controls on spending such as social programs led to significant savings. Domestic interest payments were, however, larger than anticipated due to the unforeseen upfront cost of converting 20-year government bonds into treasury bills.

4. Monetary policy has been appropriate. Targets for reserve money and net international reserves for March 2010 were met with margins. Interest rates on three-month government securities declined to 2.4 percent in May 2010. The rapid reduction of domestic debt left commercial banks with significant excess reserves despite increased central bank sterilization. Relatively low central bank rates, ample liquidity conditions, and signs of economic recovery did not boost credit growth significantly, partly reflecting high lending rates and limited competition among banks.

Main Quantitative Performance Criteria Under the EFF, December 2009 - March 2010 (end-of-period)

Net international reserves of the CBS, millions of U.S. dollars (floor)

Reserve money, millions of Seychelles rupees (ceiling)

Fiscal primary balance, millions of Seychelles rupees (cumulative floor)

$\frac{2009}{\text { December }} \frac{2010}{\text { Program Adjusted Actual }} \frac{\text { March }}{\text { Program Adjusted Actual }}$

5. The banking system is well capitalized and profitable (Table 5). Although credit quality worsened marginally, banks remain fully provisioned under current standards. They 
have three years to comply with the doubling of the minimum capital requirement (from SR 10 million to SR 20 million) of December 29, 2009.

6. Structural reforms have progressed satisfactorily (Table 8). Following their Cabinet approval on March 17 (end-March benchmark), the customs reform strategy and implementation plan are at the implementation stage, although submission of the new Customs Management Act to parliament (end-June benchmark) is delayed to endSeptember 2010 since more time was required to adjust the initial draft to the needs of a small island economy. General government finance statistics were published on April 23 (end-April benchmark). The rest of the structural reform agenda was implemented as scheduled, except for delays in initiating the privatization of state-owned banks.

7. Public external debt restructuring has advanced further. To date, the authorities reached agreements with external creditors covering around 85 percent of the debt eligible for restructuring (SMEFP 937 ). Reflecting recent agreements, the external debt stock declined from 92 percent of GDP in 2009 to 54 percent in March 2010. ${ }^{1}$ Since the successful completion of the commercial debt exchange offer in February 2010, discussions with nonParis Club bilateral and commercial creditors have also progressed. All but one bilateral creditor and one commercial creditor have either signed agreements or otherwise indicated their willingness to accept terms at least as concessional as those of the Paris Club.

Discussions with remaining creditors are ongoing.

\section{Program For the Rest Of 2010}

\section{A. Macroeconomic Framework}

8. The outlook is favorable. With better than expected growth in 2009 , the projected real GDP growth rates of 4 percent for 2010 and 5 percent for 2011 and 2012 are within reach. End-year inflation is expected to be contained to low single digits. The current account deficit will widen to 39 percent of GDP in 2010 owing to the recovery of domestic demand, resumption of FDI, and rising costs of insurance premiums because of the piracy threat, but will gradually narrow thereafter as exports of tourism services will benefit from the global economic recovery and additional hotel accommodations. Official external reserves are projected to reach 2.2 months of imports at end-2010.

\section{B. Fiscal Policy}

\section{Higher revenue projections ensure the targeted public debt reduction and create space for financing additional priority expenditure. Revenue projections for 2010 were}

\footnotetext{
${ }^{1}$ This represents a stronger reduction in the external debt stock than projected in the most recent Debt Sustainability Analysis (Country Report No. 10/3, January 5, 2010) mainly on account of the cancellation of a large late payment penalty related to the Eurobond debt exchange.
} 
revised upwards by SR 124 million or 1.1 percent of GDP as a result of the strong first quarter outcome and higher dividends expected from public enterprises. About half the additional revenue is to be saved, leading to an upwards revision of the 2010 primary balance target by SR 62 million to 7.4 percent of GDP. Savings will also be generated from faster reimbursement of government loans by two public enterprises. Additional budget appropriations mainly will finance higher-than-anticipated costs related to the introduction of a new wage grid and scheme of service for health and education (designed to reverse the loss of qualified professionals to the private sector) and termination fees for outsourced noncore workers in budgetdependent agencies. Revenue overperformance allows for the phased release of the contingency, leading to an increase in expenditure on muchneeded infrastructure investment

\begin{tabular}{lrrrr}
\multicolumn{5}{c}{ Revisions to Fiscal Targets } \\
\hline & 2010 & \multicolumn{3}{c}{ Diff. } \\
(Millions of Seychelles rupees) & Prog. & Proj. & SR m\% GDP ${ }^{1}$ \\
\hline Total revenue and grants & $\mathbf{3 , 7 2 3}$ & $\mathbf{3 , 8 6 6}$ & $\mathbf{1 4 3}$ & $\mathbf{1 . 3}$ \\
$\quad$ Total revenue & 3,390 & 3,514 & 124 & 1.1 \\
$\quad 3,067$ & 3,090 & 23 & 0.2 \\
$\quad$ Tax & 323 & 424 & 101 & 0.9 \\
$\quad$ Nontax & 333 & 352 & 19 & 0.2 \\
External grants & & & & \\
& $\mathbf{2 , 9 5 2}$ & $\mathbf{3 , 0 3 3}$ & $\mathbf{8 1}$ & $\mathbf{0 . 7}$ \\
Primary expenditure and net lending & 2,415 & 2,489 & 74 & 0.7 \\
Primary current expenditure & 867 & 893 & 26 & 0.2 \\
Capital expenditure ${ }^{2}$ & -330 & -349 & -19 & -0.2 \\
Net lending & $\mathbf{7 7 1}$ & $\mathbf{8 3 3}$ & $\mathbf{6 2}$ & $\mathbf{0 . 6}$ \\
Primary balance & 7.0 & 7.4 & $\ldots$ & $\ldots$ \\
\% of GDP & 11,057 & 11,206 & $\ldots$ & $\ldots$ \\
Nominal GDP & & & & \\
\hline
\end{tabular}

Source: Ministry of Finance and IMF estimates and projections.

${ }^{1}$ As a share of projected GDP

${ }^{2}$ Includes the release of the contingency (SMEFP ๆ12).

10. Tax reforms are on track (SMEFP $\$ 13$ ). Revised business tax legislation was enacted in January 2010, reducing rates and broadening the tax base. The Goods and Services Tax (GST) base was expanded, cascading effects were eliminated, and concessional rates are being phased out. The introduction of a withholding-based personal income tax to replace the current system of social security contributions is on track for July 2010. The authorities have hired an external expert to help them replace the current multiple-rate GST with VAT in 2012; the EU will help finance technical assistance during the implementation phase.

11. Public financial management will be further improved. Expenditure monitoring, facilitated by the Treasury Single Account set up in late 2009, will be further strengthened with ongoing tightening of internal controls and the new chart of accounts planned for 2011 (SMEFP -16). Starting with the 2011 cycle, the government is also reinforcing budget processes ( $(17)$ and preparing comprehensive budget documentation ( $\mid 18)$. Comprehensive financial and management assessments of the Social Security and Pension Funds will be undertaken with the assistance of the World Bank.

\section{Monetary, Exchange Rate, and Financial Sector Policies}

\section{A prudent monetary policy, based on reserve money targeting, remains key to} anchoring inflation expectations. As monthly inflation turns positive (reflecting the recent 
exchange rate depreciation against the dollar), reserve money targets continue to be appropriate. The CBS will continue to rely on repurchase agreements and deposit and credit auctions to achieve its targets and guide short-term rates.

13. The authorities will pursue efforts to strengthen liquidity management. Excess reserves of banks (on average 7 percent of rupee deposits over the last six months, and 3 percent of GDP) may undermine interbank market development and shift banks' focus on foreign exchange transactions. Last-minute changes in the volume of T-bill auctions create uncertainty, further complicating banks' ability to manage liquidity and weakening interest rate pass-through. To address this, the T-bill auction calendar will be announced weekly for the next four weeks, with information on volumes disclosed three days before each auction (SMEFP ๆ19). The CBS will also improve liquidity forecasting.
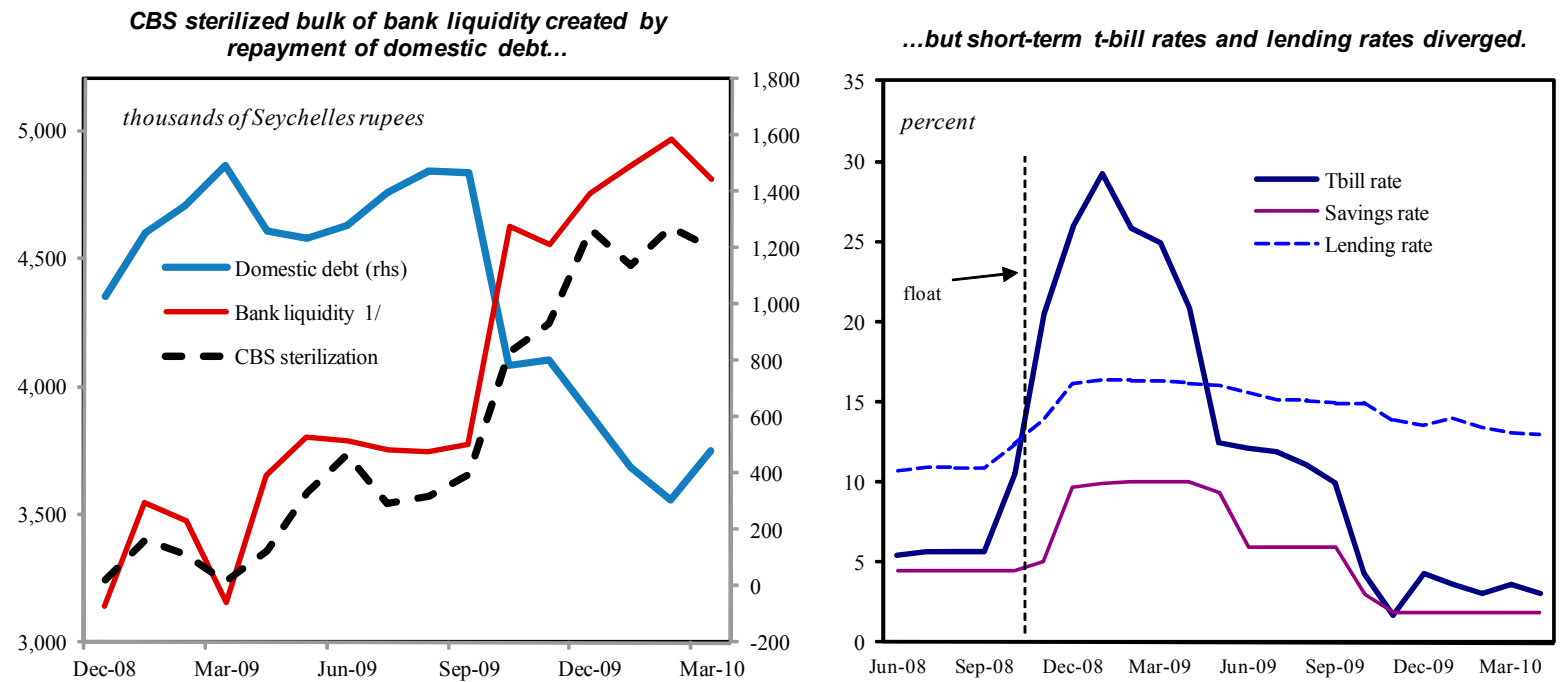

Source: Seychelles authorities and Fund staff estimates.

1/ Bank liquidity is excess reserves plus CBS sterilization

14. The authorities intend to maintain a flexible exchange rate regime, which has played a key role in adjusting to shocks since the start of the reform program. They do not intend to intervene in the foreign exchange market except for smoothing out excessive volatility (SMEFP q25).

\section{A direct securitized injection will replenish the CBS' capital to statutory levels} ( 927$)$ in 2010. The injection of T-bills amounting to 1.7 percent of GDP in three equal quarterly installments will strengthen the central bank balance sheet and enhance its portfolio of tradable securities for open market operations.

16. Other central bank operations are undergoing extensive modernization with the Fund technical assistance. The CBS promptly reformed monetary operations, banking supervision, reserve management and internal procedures and controls. Key priorities will 
now be to adopt risk-based banking supervision and best supervisory practices (SMEFP q3132 ); implement integrated (core) banking to improve efficiency and minimize operational risks ( $\mid 26)$; strengthen reserve management capabilities (with World Bank and Bank for International Settlement assistance) ( $\mid 28)$; and develop business continuity and crisis preparedness plans $(\boldsymbol{\Phi} 30)$.

\section{The authorities are assessing the timing for and modalities of the divestiture of} publicly-owned financial institutions, with the assistance of the World Bank Group. The government invited IFC to conduct a detailed analysis of Nouvobanq and asked FIRST to help determine the optimal strategy for the Development Bank of Seychelles and the Housing Finance Corporation (SMEFP \42). Meanwhile, both are under CBS regulatory oversight and contracts for comprehensive financial audits were tendered in April 2010.

\section{Public Enterprise Sector Reforms}

18. The authorities intend to put in place a comprehensive monitoring of the public enterprise sector and address the large quasi-fiscal risks. Although risks stemming from Air Seychelles and the Public Utilities Company (PUC) are currently contained, both entities should be closely monitored. An international consulting firm was engaged to carry out a strategic assessment of the national airline (SMEFP $q 41$ ); a study of the optimal tariff policy will be commissioned and PUC has been subjected to an independent operational audit (\19). To enhance transparency and governance, the Ministry of Finance also has submitted the financial audits of several public enterprises to the scrutiny of the Public Accounts Committee of the National Assembly (\$41).

\section{Program Financing, Design, AND Risks}

19. The program for $\mathbf{2 0 1 0}$ is financed. The commercial debt exchange and recent bilateral agreements with commercial banks and one bilateral creditor have significantly reduced program financing needs. The residual financing gap for 2010 is expected to be closed by restructuring the rest of public external debt.

20. Primary surplus targets (quantitative performance criteria) for June, September and December 2010 have been revised upwards to reflect updated revenue projections. The new targets lock in half the additional revenue.

21. The CBS was subject to an update safeguards assessment in the context of the new arrangement. The assessment, which is substantially complete, has found continued improvement in the safeguards framework of the central bank. The bank's 2009 financial statements were prepared and audited in accordance with international standards and have been published, and governance oversight has been strengthened. Nevertheless, some vulnerabilities remain concerning internal audit and controls, and the legal framework, which the authorities are committed to addressing. The recommendations in the draft assessment 
report do not require action in the context of the first review and will be closely monitored going forward.

22. The main risks to the outlook and the program stem from the volatile external environment and the run-up to the 2011 presidential elections. A protracted slowdown in Europe - the main source market for tourism - and further depreciation of the euro would undermine export proceeds and economic activity. Piracy in the Indian Ocean could impinge upon tourism and fishing. Political opposition and vested interests could slow tax reforms and public enterprise restructuring. These risks appear manageable in view of the strong program ownership and track record of policy adjustment to external shocks under the previous arrangement, ongoing international anti-piracy efforts, and broad political support for the reforms.

\section{StafF ApPRAisal}

23. Seychelles has made a good start on its second stage of reforms under an EFFsupported program, despite a difficult international environment, showing strong resilience to the double crisis it confronted. Less than two years into a spectacular turnaround in its economic policies to address an external debt crisis and a global crisis, the country appears to have emerged from the recession earlier than initially expected.

\section{The economy is reaping the benefits of strong macroeconomic stabilization} policies. The continuation of prudent fiscal and monetary policies has granted credibility to the currency float introduced in November 2008; inflation has virtually disappeared; international reserves are being rebuilt, and key policy interest rates have been reduced.

25. Seychelles remains highly exposed to external shocks. Because piracy threats, risks to the European economic outlook (by far the main market for tourism), and further depreciation of the euro may affect the country's still fragile external position, the authorities should continue to stand ready to adjust policies as needed. Their decision to save half the upward revision in government revenue projected in 2010 is commendable. These savings will secure the targeted government debt reduction and contribute to strengthening the external position despite an adverse global environment.

\section{Progress on the ambitious program of tax and public finance management} reforms is encouraging, but important steps still lie ahead. The imminent replacement of the social security contribution by the PIT is an opportunity to eliminate concessional rates, broaden the tax base, and reduce tax rates. The introduction of VAT in 2012 will further eliminate cascading effects of the existing sales tax and set the proper incentives for exporters. The modernization of customs is an important step toward establishing a businessfriendly environment. The authorities are also encouraged to further improve the budget preparation and execution cycle. 
27. Seychelles has made an excellent use of the Fund technical assistance, particularly in the modernization of central bank operations. Progress in ensuring an independent monetary policy, strengthening supervision, and implementing the Fund's safeguard assessment recommendations is commendable. Further coordination with the treasury on the issuance of T-bills is needed to improve liquidity management and provide a more predictable environment for banks, facilitating the transmission of monetary policy signals.

28. Restructuring the public enterprise sector is an essential condition to achieving high medium-term growth, driven by the development of a competitive private sector. The recommendations of the ongoing financial and management audits for all key public enterprises should be implemented timely. Privatization should be actively pursued when market conditions permit, including for state-owned financial institutions. Staff welcomes the launch of preparatory work to put the public utilities company on a sound footing (including through proper tariff policies), to reform the social security institutions, and to establish an actuarially sound pension system.

29. Staff encourages the authorities to complete the discussions on the restructuring of its external debt while ensuring strict comparability of treatment among creditors. The strong track record of policy implementation has convinced most external creditors to bring back external debt to sustainable levels, including through successful debt exchange with private creditors.

30. In light of strong performance through end-April 2010, compliance with the performance criteria, consistency with the Fund's Lending into Arrears policy, and satisfactory update safeguards assessment, staff recommends completion of the first EFF arrangement program and of financing assurances reviews. Staff supports the modification of the quantitative performance criterion on the fiscal primary balance for endJune, end-September, and end-December 2010. 
Table 1. Seychelles: Selected Economic and Financial Indicators, 2008-12

\begin{tabular}{|c|c|c|c|c|c|c|c|}
\hline & \multirow[t]{2}{*}{2008} & \multicolumn{2}{|c|}{2009} & \multicolumn{2}{|c|}{2010} & \multirow{2}{*}{$\begin{array}{c}2011 \\
\text { Proj. }\end{array}$} & \multirow{2}{*}{$\begin{array}{c}2012 \\
\text { Proj. }\end{array}$} \\
\hline & & Prog. & Prel. & Prog. & Proj. & & \\
\hline National income and prices ${ }^{1}$ & \multicolumn{7}{|c|}{ (Percentage change, unless otherwise indicated) } \\
\hline Nominal GDP (millions of Seychelles rupees) & 8,710 & 10,402 & 10,726 & 11,057 & 11,206 & 12,091 & 13,059 \\
\hline Real GDP & -1.3 & -7.6 & 0.7 & 4.0 & 4.0 & 5.0 & 5.0 \\
\hline CPI (annual average) & 37.0 & 32.9 & 31.8 & 2.3 & -2.4 & 2.5 & 3.1 \\
\hline CPI (end-of-period) & 63.3 & 1.9 & -2.5 & 2.2 & 1.1 & 2.9 & 3.0 \\
\hline GDP deflator average & 29.3 & 28.5 & 22.3 & 2.2 & 0.4 & 2.8 & 2.9 \\
\hline Money and credit & \multicolumn{7}{|c|}{ (Percentage change, unless otherwise indicated) } \\
\hline Net claims on private sector & 48.6 & -12.1 & -11.4 & 27.3 & 22.2 & $\ldots$ & $\ldots$ \\
\hline Broad money & 27.2 & -0.7 & 7.5 & 25.6 & 19.1 & $\ldots$ & $\ldots$ \\
\hline Reserve money & 0.6 & 32.2 & 15.7 & 18.4 & 35.3 & $\ldots$ & $\ldots$ \\
\hline Velocity (GDP/broad money) & 1.5 & 1.8 & 1.7 & 1.5 & 1.5 & $\ldots$ & $\ldots$ \\
\hline Money multiplier (broad money/reserve money) & 5.3 & 4.0 & 4.9 & 4.2 & 4.3 & $\ldots$ & $\ldots$ \\
\hline Savings-Investment balance & \multicolumn{7}{|c|}{ (In percent of GDP) } \\
\hline External savings & 51.8 & 22.6 & 35.0 & 32.7 & 39.5 & 35.5 & 30.2 \\
\hline Gross national savings & -11.7 & 6.8 & -5.8 & -0.9 & 6.0 & 11.9 & 13.3 \\
\hline Of which: government savings & 7.9 & 2.8 & 7.8 & 2.7 & 5.6 & 9.2 & 9.3 \\
\hline Gross investment & 40.1 & 29.4 & 29.3 & 31.8 & 45.4 & 47.3 & 43.5 \\
\hline Of which: government investment & 2.1 & 5.3 & 5.7 & 6.8 & 8.0 & 7.3 & 8.5 \\
\hline \multicolumn{8}{|l|}{ Government budget } \\
\hline Total revenue, excluding grants & 32.9 & 35.6 & 35.4 & 30.7 & 31.4 & 31.2 & 31.0 \\
\hline Expenditure and net lending & 40.0 & 35.1 & 33.8 & 32.8 & 33.8 & 31.3 & 32.3 \\
\hline Current expenditure & 28.7 & 32.8 & 31.0 & 28.0 & 28.9 & 24.2 & 23.8 \\
\hline Capital expenditure and net lending ${ }^{1}$ & 11.2 & 2.3 & 2.7 & 4.9 & 4.9 & 7.1 & 8.5 \\
\hline Overall balance, including grants & -3.4 & 2.8 & 5.0 & 0.9 & 0.7 & 2.1 & 0.8 \\
\hline Primary balance & 3.9 & 13.3 & 14.8 & 7.0 & 7.4 & 6.0 & 4.7 \\
\hline Total public debt ${ }^{2,3}$ & 136.7 & 140.1 & 128.2 & 109.5 & 82.4 & 76.7 & 65.4 \\
\hline Domestic & 53.9 & 41.6 & 36.0 & 37.4 & 28.8 & 23.6 & 19.0 \\
\hline External 2,3 & 82.8 & 98.5 & 92.2 & 72.2 & 53.6 & 53.1 & 46.4 \\
\hline External sector & \multicolumn{7}{|c|}{ (In percent of GDP, unless otherwise indicated) } \\
\hline Current account balance including official transfers & -51.8 & -22.6 & -35.0 & -32.7 & -39.5 & -35.5 & -30.2 \\
\hline Total stock of arrears (millions of U.S. dollars) ${ }^{2,3}$ & 317 & 239 & 244 & 231 & 86 & 86 & 86 \\
\hline Total public external debt outstanding (millions of U.S. dollars) 2, $3^{2}$ & 759 & 755 & 729 & 708 & 492 & 516 & 551 \\
\hline (in percent of GDP) & 82.8 & 98.5 & 92.2 & 72.2 & 53.6 & 52.9 & 53.4 \\
\hline Terms of trade (= - deterioration) & 0.7 & -2.1 & -3.2 & $\ldots$ & $\ldots$ & $\ldots$ & $\ldots$ \\
\hline Real effective exchange rate (average, percent change) & -7.1 & $\ldots$ & -6.6 & $\ldots$ & $\ldots$ & $\ldots$ & $\ldots$ \\
\hline Gross official reserves (end of year, millions of U.S. dollars) & 51 & 153 & 169 & 202 & 224 & 274 & 324 \\
\hline In months of imports, c.i.f. & 0.6 & 1.5 & 1.7 & 1.9 & 2.3 & 2.7 & 3.0 \\
\hline \multicolumn{8}{|l|}{ Exchange rate } \\
\hline Seychelles rupees per US $\$ 1$ (end of period) & 16.6 & 10.6 & 11.3 & $\ldots$ & $\ldots$ & $\ldots$ & $\ldots$ \\
\hline Seychelles rupees per US $\$ 1$ (period average) & 9.5 & 14.1 & 13.6 & $\ldots$ & $\ldots$ & $\ldots$ & $\ldots$ \\
\hline
\end{tabular}

Sources: Central Bank of Seychelles; Ministry of Finance; and IMF staff estimates and projections.

${ }^{1}$ In 2010 includes contingency spending.

${ }^{2}$ Reflects the impact of agreed debt restructuring including the Paris Club debt agreement of May 2009, and the commercial debt exchange in 2010 Q1.

${ }^{3}$ Includes arrears and the external debt of the central bank. 
Table 2. Seychelles: Balance of Payments, 2008-12

(Millions of U.S. dollars)

\begin{tabular}{|c|c|c|c|c|c|c|c|c|c|c|c|c|c|c|}
\hline & \multirow{3}{*}{$\begin{array}{l}2008 \\
\text { Prel. }\end{array}$} & \multirow{3}{*}{$\begin{array}{c}2009 \\
\text { Prel. }\end{array}$} & \multicolumn{10}{|c|}{2010} & \multirow{3}{*}{$\begin{array}{c}2011 \\
\text { Year } \\
\text { Proj. }\end{array}$} & \multirow{3}{*}{$\begin{array}{c}2012 \\
\text { Year } \\
\text { Proj. }\end{array}$} \\
\hline & & & \multicolumn{2}{|c|}{ Q1 } & \multicolumn{2}{|c|}{ Q2 } & \multicolumn{2}{|c|}{ Q3 } & \multicolumn{2}{|c|}{ Q4 } & \multicolumn{2}{|c|}{ Year } & & \\
\hline & & & Prog. & Prel. & Prog. & Proj. & Prog. & Proj. & Prog. & Proj. & Prog. & Proj. & & \\
\hline Current account & -475 & -277 & -56 & -21 & -51 & -85 & -40 & -116 & -173 & -156 & -321 & -362 & -346 & -312 \\
\hline (Percent of GDP) & -52 & -35 & -6 & -2 & -5 & -9 & -4 & -13 & -18 & -17 & -33 & -39 & -35 & -30 \\
\hline Trade balance & -412 & -228 & -52 & -27 & -31 & -58 & -27 & -94 & -152 & -137 & -263 & -301 & -278 & -252 \\
\hline Exports of goods & 502 & 427 & 129 & 139 & 119 & 107 & 149 & 115 & 143 & 100 & 540 & 460 & 455 & 466 \\
\hline Of which: oil re-exports & 258 & 149 & 73 & 55 & 68 & 39 & 75 & 50 & 76 & 62 & 292 & 206 & 222 & 235 \\
\hline Of which: tuna exports & 214 & 165 & 47 & 58 & 37 & 51 & 61 & 48 & 58 & 25 & 203 & 181 & 200 & 195 \\
\hline Imports of goods & $-1,009$ & -748 & -213 & -190 & -178 & -177 & -190 & -222 & -297 & -237 & -878 & -826 & -845 & -867 \\
\hline of which: Oil imports & -364 & -203 & -88 & -66 & -78 & -50 & -83 & -63 & -84 & -70 & -333 & -249 & -252 & -275 \\
\hline FDI-related imports & -288 & -176 & -41 & -24 & -45 & -38 & -49 & -57 & -68 & -57 & -204 & -177 & -182 & -147 \\
\hline Other & -352 & -353 & -83 & -100 & -54 & -89 & -59 & -101 & -144 & -111 & -341 & -399 & -411 & -445 \\
\hline Exports of services & 486 & 398 & 112 & 107 & 109 & 93 & 120 & 108 & 121 & 102 & 462 & 410 & 446 & 497 \\
\hline Of which: tourism earnings & 270 & 205 & 61 & 65 & 56 & 47 & 64 & 56 & 69 & 47 & 250 & 215 & 250 & 290 \\
\hline Imports of services & -391 & -305 & -81 & -84 & -81 & -81 & -106 & -95 & -120 & -102 & -387 & -346 & -333 & -349 \\
\hline Income, net & -120 & -91 & -18 & -2 & -30 & -28 & -25 & -22 & -33 & -21 & -106 & -74 & -72 & -63 \\
\hline Of which: transfers of profits and dividends & -51 & -47 & -15 & -8 & -15 & -7 & -20 & -13 & -17 & -9 & -67 & -37 & -42 & -34 \\
\hline Of which: interest payments due ${ }^{1,3}$ & -70 & -48 & -2 & -3 & -14 & -21 & -2 & -7 & -15 & -7 & -32 & -41 & -38 & -38 \\
\hline of which: Public sector ${ }^{2}$ & -33 & -37 & -2 & 0 & -14 & -19 & -2 & -3 & -15 & -3 & -32 & -29 & -14 & -18 \\
\hline Current transfers, net & 57 & 41 & 15 & 9 & 10 & 1 & 12 & 0 & 12 & 2 & 48 & 12 & 4 & 4 \\
\hline of which: General government, net & 32 & 39 & 10 & 10 & 2 & 0 & 4 & 0 & 1 & 3 & 17 & 13 & 15 & 15 \\
\hline Capital and financial account & 366 & 121 & 58 & 70 & 30 & 45 & 57 & 117 & 144 & 153 & 291 & 385 & 374 & 355 \\
\hline Capital account 4,5 & 5 & 56 & 4 & 234 & 4 & 6 & 46 & 57 & 8 & 7 & 62 & 304 & 0 & 17 \\
\hline Financial account & 361 & 64 & 54 & -164 & 26 & 40 & 11 & 59 & 136 & 146 & 229 & 80 & 374 & 338 \\
\hline Direct investment, net ${ }^{3}$ & 166 & 113 & 50 & 26 & 54 & 26 & 57 & 40 & 84 & 40 & 245 & 133 & 126 & 100 \\
\hline Portfolio investment, net ${ }^{2}$ & 125 & 1 & 0 & 0 & 0 & 0 & 0 & 0 & 0 & 0 & 1 & 1 & 1 & 1 \\
\hline Other investment, net & 70 & -50 & 4 & -191 & -29 & 13 & -46 & 19 & 52 & 106 & -17 & -53 & 247 & 237 \\
\hline Of which: Government and government-guaranteed & 19 & -110 & -12 & -236 & -14 & -8 & -42 & -37 & 11 & 11 & -55 & -270 & 12 & 32 \\
\hline Disbursements & 84 & 12 & 1 & 9 & 1 & 3 & 9 & 12 & 24 & 13 & 36 & 37 & 30 & 40 \\
\hline Project loans & 84 & 1 & 1 & 1 & 1 & 3 & 9 & 3 & 4 & 3 & 16 & 10 & 10 & 20 \\
\hline Program loans & 0 & 11 & 0 & 9 & 0 & 0 & 0 & 9 & 20 & 10 & 20 & 27 & 20 & 20 \\
\hline Amortization (Principal payments) ${ }^{2}$ & -65 & -79 & -13 & -13 & -16 & -11 & -13 & -9 & -13 & -2 & -52 & -35 & -16 & -7 \\
\hline Debt forgiveness 5 & $\ldots$ & -45 & 0 & -231 & 0 & 0 & -39 & -39 & 0 & 0 & -39 & -270 & 0 & 0 \\
\hline Of which: Other liabilities & 54 & 89 & 4 & 33 & -10 & 25 & 6 & 66 & 22 & 76 & 22 & 201 & 225 & 201 \\
\hline of which: foreign loans for investment ${ }^{3}$ & 185 & 110 & 6 & 26 & -9 & 26 & 8 & 38 & 23 & 38 & 25 & 128 & 121 & 98 \\
\hline Net errors and omissions & -102 & 133 & 0 & -29 & 0 & 0 & 0 & 0 & 0 & 0 & 0 & -29 & 0 & 0 \\
\hline Overall balance & -212 & -24 & 2 & 20 & -22 & -40 & 17 & 1 & -29 & -3 & -30 & -7 & 28 & 44 \\
\hline Financing & 212 & 24 & -1 & -20 & 5 & 25 & -14 & -11 & -29 & -14 & -39 & -21 & -43 & -50 \\
\hline Change in net international reserves (increase: -) & 38 & -43 & -1 & -41 & 5 & 25 & -14 & -11 & -29 & -14 & -39 & -42 & -43 & -50 \\
\hline Change in gross official reserves (increase: - ) & 28 & -50 & -5 & -41 & 2 & 18 & -18 & -14 & -29 & -17 & -49 & -54 & -50 & -50 \\
\hline Liabilities to IMF, net & 10 & 8 & 3 & 0 & 3 & 7 & 3 & 3 & 0 & 3 & 10 & 12 & 7 & 0 \\
\hline Other Net Foreign Assets (in & 0 & -13 & 0 & 10 & 0 & 0 & 0 & 0 & 0 & 0 & 0 & 10 & 0 & 0 \\
\hline Exceptional financing & 174 & 79 & 0 & 11 & 0 & 0 & 0 & 0 & 0 & 0 & 0 & 11 & 0 & 0 \\
\hline Change in arrears (increase: + ) ${ }^{4,6}$ & 174 & -74 & 0 & -159 & 0 & 0 & 0 & 0 & 0 & 0 & 0 & -159 & 0 & 0 \\
\hline Clearance of arrears & 0 & 153 & 0 & 170 & 0 & 0 & 0 & 0 & 0 & 0 & 0 & 170 & 0 & 0 \\
\hline Financing gap & 0 & 0 & 0 & 0 & 16 & 15 & -2 & 10 & 57 & 18 & 69 & 28 & 14 & 6 \\
\hline Memorandum items: & & & & & & & & & & & & & & \\
\hline Exports growth, percent & 28.1 & -14.9 & 23.5 & 34.2 & 24.3 & 23.6 & 25.6 & -6.5 & 30.8 & -12.7 & 26.1 & 7.8 & -1.2 & 2.4 \\
\hline Tourism growth, percent & -4.8 & -24.3 & 3.4 & 10.7 & 21.5 & 12.2 & -7.2 & 6.5 & 7.2 & -8.8 & 4.9 & 5.0 & 16.2 & 16.2 \\
\hline Imports growth, percent & 37.5 & -25.9 & 24.6 & 13.9 & 17.6 & 12.8 & 22.8 & 11.4 & 28.7 & 5.3 & 24.0 & 10.4 & 2.4 & 2.5 \\
\hline o/w non-FDI and domestic-oil imports & 13.3 & -11.2 & $\ldots$ & & & & & & $\ldots$ & & & 3.9 & 4.9 & 9.3 \\
\hline Exports, percent of GDP & 54.8 & 54.0 & 0.0 & 0.0 & 0.0 & 12.8 & 0.0 & 11.4 & 0.0 & 5.3 & 45.6 & 40.2 & 46.6 & 45.1 \\
\hline Imports, percent of GDP & 110.1 & 94.6 & 0.0 & 0.0 & 0.0 & 0.0 & 0.0 & 0.0 & 0.0 & 0.0 & 74.1 & 72.1 & 86.7 & 84.0 \\
\hline FDI (incl. loans), USD million ${ }^{3}$ & 350 & 223 & 56 & 52 & 45 & 52 & 65 & 78 & 107 & 78 & 270 & 260 & 247 & 198 \\
\hline FDI (incl. loans), percent of GDP & 38.2 & 28.2 & $\ldots$ & $\ldots$ & $\ldots$ & $\ldots$ & $\ldots$ & $\ldots$ & $\ldots$ & $\ldots$ & 25.0 & 28.3 & 25.3 & 19.2 \\
\hline Gross international reserves (stock, e.o & 51 & 169 & 158 & 210 & 156 & 192 & 174 & 207 & 202 & 224 & 202 & 224 & 274 & 324 \\
\hline Months of prospective imports of goods and services & 0.6 & 1.7 & 1.5 & 2.1 & 1.5 & 2.0 & 1.6 & 2.1 & 1.9 & 2.3 & 1.9 & 2.3 & 2.7 & 3.0 \\
\hline o.w. Valuation change (+gain) & & 68 & & & & & & & & & & & & \\
\hline & 221 & 125 & 16 & 17 & 29 & 32 & 15 & 17 & 27 & 8 & 86 & 78 & 54 & 46 \\
\hline (Percent of exports of goods and services) & 22.41 & 15.13 & 6.64 & 6.90 & 12.88 & 16.17 & 5.68 & 7.59 & 10.20 & 4.10 & 8.54 & 8.90 & 6.04 & 4.73 \\
\hline Public external debt ${ }^{1,5}$ & 759 & 729 & $\ldots$ & 504 & $\ldots$ & & $\ldots$ & ... & $\ldots$ & .. & 708 & 492 & 516 & 551 \\
\hline (Percent of GDP) & 82.8 & 92.2 & & 54.8 & & & & & $\begin{array}{l}\cdots \\
\cdots\end{array}$ & & 72.2 & 53.6 & 52.9 & 53.4 \\
\hline GDP & 916 & 790 & 981 & 919 & 981 & 919 & 981 & 919 & 981 & 919 & 981 & 919 & 975 & 1,032 \\
\hline
\end{tabular}

Sources: Central Bank of Seychelles; Ministry of Finance; and IMF staff estimates and projections.

${ }^{1}$ Reflects the restructuring with Paris Club (with exception of a mortgaged oil tanker); three non-Paris Club creditors (South Africa, Malaysia, and Libya); and the commercial debt exchange (which included amortizing notes, bond, a commercial bank, and a land settlement) and some other commercial debt. The Paris Club agreement includes deferral of some interest during 2008 and June 2009.

${ }^{2}$ The eurobonds and amortizing notes as well as the bonds issued after the commercial debt exchange were classified as portfolio flows instead of other investment loans.

${ }^{3}$ Reclassification of FDI. Preliminary findings suggest that a significant portion of FDI is foreign loans from non-parents.

${ }^{4}$ Debt forgiveness contributed to the sharp increase in capital inflows in 2009 and 2010.

${ }^{5}$ Debt forgiveness for Paris Club and some non-Paris Club creditors is two-stage (in 2009 and 2010).

${ }^{6}$ In 2008, includes accelerated promissory notes.

${ }^{7}$ Refers to CBS gross international reserves net of blocked deposits and project accounts. 
Table 3. Seychelles: Consolidated Government Operations, 2008-2012 ${ }^{1}$

(Millions of Seychelles rupees; cumulative from the start of the year)

\begin{tabular}{|c|c|c|c|c|c|c|c|c|c|c|c|c|}
\hline & \multirow[t]{3}{*}{2008} & \multirow[t]{3}{*}{2009} & \multicolumn{8}{|c|}{2010} & \multirow{3}{*}{$\begin{array}{r}2011 \\
\text { Proj. }\end{array}$} & \multirow{3}{*}{$\begin{array}{r}2012 \\
\text { Proj. }\end{array}$} \\
\hline & & & \multicolumn{2}{|c|}{ Q1 } & \multicolumn{2}{|c|}{ Q2 } & \multicolumn{2}{|c|}{ Q3 } & \multicolumn{2}{|c|}{ Q4 } & & \\
\hline & & & Prog. & Prel. & Prog. & Proj. & Prog. & Proj. & Prog. & Proj. & & \\
\hline Total revenue and grants & 3,190 & 4,165 & 835 & 848 & 1,679 & 1,796 & 2,685 & 2,829 & 3,723 & 3,866 & 4,039 & 4,330 \\
\hline Total revenue & 2,868 & 3,793 & 776 & 822 & 1,559 & 1,694 & 2,444 & 2,576 & 3,390 & 3,514 & 3,778 & 4,048 \\
\hline Tax & 2,456 & 3,318 & 712 & 747 & 1,425 & 1,513 & 2,222 & 2,265 & 3,067 & 3,090 & 3,376 & 3,622 \\
\hline Personal Income Tax & & 0 & 0 & 0 & 0 & 0 & 79 & 69 & 197 & 190 & 450 & 486 \\
\hline Social security tax & 295 & 384 & 109 & 105 & 201 & 200 & 230 & 233 & 230 & 233 & 0 & 0 \\
\hline Trade tax & 410 & 437 & 104 & 145 & 210 & 297 & 333 & 444 & 491 & 620 & 669 & 723 \\
\hline Goods and services tax (GST) & 951 & 1,349 & 284 & 258 & 603 & 565 & 922 & 832 & 1,282 & 1,160 & 1,290 & 1,410 \\
\hline Business tax & 524 & 802 & 136 & 126 & 268 & 284 & 454 & 467 & 599 & 602 & 649 & 666 \\
\hline Other & 277 & 346 & 80 & 112 & 143 & 167 & 204 & 220 & 269 & 286 & 318 & 337 \\
\hline Nontax & 412 & 474 & 64 & 75 & 133 & 181 & 222 & 311 & 323 & 424 & 401 & 426 \\
\hline Fees and charges & 145 & 182 & 44 & 55 & 87 & 101 & 131 & 148 & 174 & 195 & 194 & 202 \\
\hline Dividends from parastatals & 64 & 148 & 10 & 8 & 30 & 31 & 59 & 87 & 99 & 129 & 104 & 112 \\
\hline Other & 203 & 143 & 10 & 12 & 17 & 50 & 32 & 76 & 50 & 101 & 103 & 111 \\
\hline External grants & 321 & 373 & 59 & 26 & 121 & 101 & 242 & 253 & 333 & 352 & 261 & 282 \\
\hline Expenditure and net lending & 3,483 & 3,625 & 670 & 767 & 1,571 & 1,849 & 2,449 & 2,826 & 3,628 & 3,788 & 3,781 & 4,220 \\
\hline Current expenditure & 2,503 & 3,329 & 655 & 690 & 1,494 & 1,679 & 2,191 & 2,502 & 3,091 & 3,244 & 2,922 & 3,113 \\
\hline $\begin{array}{l}\text { Primary current expenditure } \\
\text { Wages and salaries }\end{array}$ & $\begin{array}{r}1,875 \\
688\end{array}$ & $\begin{array}{r}2,283 \\
766\end{array}$ & $\begin{array}{l}556 \\
176\end{array}$ & $\begin{array}{l}473 \\
158\end{array}$ & $\begin{array}{r}1,162 \\
352\end{array}$ & $\begin{array}{r}1,166 \\
347\end{array}$ & $\begin{array}{r}1,760 \\
528\end{array}$ & $\begin{array}{r}1,831 \\
541\end{array}$ & $\begin{array}{r}2,415 \\
704\end{array}$ & $\begin{array}{r}2,489 \\
735\end{array}$ & $\begin{array}{r}2,457 \\
717\end{array}$ & $\begin{array}{r}2,612 \\
775\end{array}$ \\
\hline Goods and services & 489 & 715 & 150 & 133 & 344 & 366 & 535 & 578 & 754 & 777 & 838 & 905 \\
\hline Transfers & 692 & 783 & 227 & 180 & 460 & 446 & 688 & 702 & 945 & 966 & 890 & 919 \\
\hline Social program of central government & 192 & 290 & 75 & 49 & 157 & 145 & 234 & 237 & 339 & 341 & 298 & 320 \\
\hline Transfers to public sector from central government & 220 & 199 & 64 & 51 & 127 & 131 & 191 & 203 & 255 & 273 & 251 & 230 \\
\hline Benefits and programs of Social Security Fund & 279 & 295 & 88 & 80 & 175 & 171 & 263 & 261 & 351 & 352 & 342 & 369 \\
\hline Other & 6 & 19 & 3 & 2 & 6 & 6 & 9 & 9 & 12 & 12 & 12 & 12 \\
\hline Interest payments due & 629 & 1,046 & 100 & 217 & 333 & 513 & 430 & 671 & 676 & 755 & 465 & 501 \\
\hline Foreign interest & 344 & 430 & 24 & 16 & 180 & 238 & 198 & 275 & 367 & 316 & 168 & 228 \\
\hline Domestic interest & 285 & 616 & 75 & 201 & 150 & 275 & 230 & 396 & 308 & 439 & 226 & 199 \\
\hline Capital expenditure & 183 & 608 & 104 & 78 & 241 & 293 & 478 & 552 & 747 & 833 & 888 & 1,107 \\
\hline Net lending & 796 & -315 & -90 & -1 & -190 & -123 & -290 & -263 & -330 & -349 & -30 & 0 \\
\hline Contingency & & 3 & 0 & 0 & 25 & 0 & 70 & 35 & 120 & 60 & 0 & 0 \\
\hline Primary balance & 336 & 1,586 & 265 & 298 & 441 & 460 & 667 & 675 & 771 & 833 & 723 & 611 \\
\hline Overall balance, commitment basis & -293 & 540 & 165 & 81 & 108 & -53 & 237 & 4 & 95 & 78 & 258 & 110 \\
\hline Change in arrears & 31 & 96 & -25 & -8 & -50 & -38 & -75 & -69 & -100 & -100 & 0 & 0 \\
\hline External interest & 167 & 321 & 0 & 0 & 0 & 0 & 0 & 0 & 0 & 0 & 0 & 0 \\
\hline Budget & -136 & -224 & -25 & -8 & -50 & -38 & -75 & -69 & -100 & -100 & 0 & 0 \\
\hline Overall balance, cash basis (after grants) & -262 & 637 & 140 & 73 & 58 & -92 & 162 & -66 & -5 & -22 & 258 & 110 \\
\hline Financing & 262 & -637 & -140 & -73 & -58 & 92 & -162 & 66 & 5 & 22 & -258 & -110 \\
\hline Foreign financing & 233 & -174 & -132 & -45 & -288 & -134 & -324 & -98 & -194 & 42 & 153 & 400 \\
\hline Disbursements & 672 & 135 & 11 & 107 & 28 & 146 & 135 & 290 & 405 & 442 & 368 & 512 \\
\hline Project loans & 672 & 14 & 11 & 8 & 28 & 46 & 135 & 83 & 181 & 118 & 123 & 253 \\
\hline Program/budget support & 0 & 120 & 0 & 99 & 0 & 99 & 0 & 206 & 224 & 324 & 245 & 259 \\
\hline Scheduled amortization & $-1,630$ & $-1,090$ & -143 & -152 & -322 & -286 & -465 & -394 & -611 & -413 & -228 & -125 \\
\hline Change in amortization arrears & 1,191 & $-1,213$ & 0 & $-1,945$ & 0 & $-1,945$ & 0 & $-1,945$ & 0 & $-1,945$ & 0 & 0 \\
\hline Clearance of arrears & 0 & 1,938 & 0 & 1,945 & 0 & 1,945 & 0 & 1,945 & 0 & 1,945 & 0 & 0 \\
\hline Debt service relief & 0 & 56 & 0 & 0 & 6 & 6 & 6 & 6 & 12 & 12 & 13 & 13 \\
\hline Domestic financing, net & -70 & -706 & -59 & -131 & 125 & -89 & -8 & -307 & -52 & -733 & -753 & -743 \\
\hline Bank financing & -100 & -823 & -53 & -140 & 112 & -40 & -7 & -173 & -46 & -494 & -715 & -706 \\
\hline CBS & -3 & -376 & -18 & -177 & 237 & -177 & 198 & -177 & 185 & -177 & -377 & -372 \\
\hline CBS recapitalization & $\ldots$ & $\ldots$ & $\ldots$ & $\ldots$ & $\ldots$ & 63 & $\ldots$ & 125 & 200 & 188 & $\ldots$ & $\ldots$ \\
\hline Commercial banks & -96 & -447 & -35 & 37 & 75 & 74 & -5 & -122 & -31 & -505 & -339 & -334 \\
\hline Nonbank financing & 30 & 117 & -6 & 9 & 12 & 13 & -1 & -9 & -5 & -51 & -38 & -37 \\
\hline Privatization and long-term lease of fixed assets & 219 & 222 & 50 & 51 & 105 & 86 & 170 & 119 & 250 & 152 & 163 & 155 \\
\hline Statistical discrepancy & -120 & 22 & 0 & 52 & 0 & 52 & 0 & 52 & 0 & 52 & 0 & 0 \\
\hline Fiscal financing gap & 0 & 0 & -42 & 0 & 44 & 177 & -21 & 298 & 465 & 510 & 179 & 78 \\
\hline \multicolumn{13}{|l|}{ Memorandum item: } \\
\hline External debt service due & 1,974 & 1,521 & 167 & 168 & 501 & 524 & 663 & 669 & 978 & 729 & 397 & 353 \\
\hline
\end{tabular}


Table 3. Seychelles: Consolidated Government Operations, $2008-2012{ }^{1}$ (continued)

(Percent of GDP; cumulative from the start of the year)

\begin{tabular}{|c|c|c|c|c|c|c|c|c|c|c|c|c|}
\hline & \multirow[t]{3}{*}{2008} & \multirow[t]{3}{*}{2009} & \multicolumn{8}{|c|}{2010} & \multirow{3}{*}{$\begin{array}{r}2011 \\
\text { Proj. }\end{array}$} & \multirow{3}{*}{$\begin{array}{r}2012 \\
\text { Proj. }\end{array}$} \\
\hline & & & \multicolumn{2}{|c|}{ Q1 } & \multicolumn{2}{|c|}{ Q2 } & \multicolumn{2}{|c|}{ Q3 } & \multicolumn{2}{|c|}{ Q4 } & & \\
\hline & & & Prog. & Prel. & Prog. & Proj. & Prog. & Proj. & Prog. & Proj. & & \\
\hline Total revenue and grants & 36.6 & 38.8 & 7.6 & 7.7 & 15.2 & 16.0 & 24.3 & 25.2 & 33.7 & 34.5 & 33.4 & 33.2 \\
\hline Total revenue & 32.9 & 35.4 & 7.0 & 7.4 & 14.1 & 15.1 & 22.1 & 23.0 & 30.7 & 31.4 & 31.2 & 31.0 \\
\hline Tax & 28.2 & 30.9 & 6.4 & 6.8 & 12.9 & 13.5 & 20.1 & 20.2 & 27.7 & 27.6 & 27.9 & 27.7 \\
\hline Personal Income Tax & & & 0.0 & 0.0 & 0.0 & 0.0 & 0.7 & 0.6 & 1.8 & 1.7 & 3.7 & 3.7 \\
\hline Social security tax & 3.4 & 3.6 & 1.0 & 1.0 & 1.8 & 1.8 & 2.1 & 2.1 & 2.1 & 2.1 & 0.0 & 0.0 \\
\hline Trade tax & 4.7 & 4.1 & 0.9 & 1.3 & 1.9 & 2.6 & 3.0 & 4.0 & 4.4 & 5.5 & 5.5 & 5.5 \\
\hline Goods and services tax (GST) & 10.9 & 12.6 & 2.6 & 2.3 & 5.4 & 5.0 & 8.3 & 7.4 & 11.6 & 10.3 & 10.7 & 10.8 \\
\hline Business tax & 6.0 & 7.5 & 1.2 & 1.1 & 2.4 & 2.5 & 4.1 & 4.2 & 5.4 & 5.4 & 5.4 & 5.1 \\
\hline Other & 3.2 & 3.2 & 0.7 & 1.0 & 1.3 & 1.5 & 1.8 & 2.0 & 2.4 & 2.6 & 2.6 & 2.6 \\
\hline Nontax & 4.7 & 4.4 & 0.6 & 0.7 & 1.2 & 1.6 & 2.0 & 2.8 & 2.9 & 3.8 & 3.3 & 3.3 \\
\hline Fees and charges & 1.7 & 1.7 & 0.4 & 0.5 & 0.8 & 0.9 & 1.2 & 1.3 & 1.6 & 1.7 & 1.6 & 1.5 \\
\hline Dividends from parastatals & 0.7 & 1.4 & 0.1 & 0.1 & 0.3 & 0.3 & 0.5 & 0.8 & 0.9 & 1.1 & 0.9 & 0.9 \\
\hline Other & 2.3 & 1.3 & 0.1 & 0.1 & 0.1 & 0.4 & 0.3 & 0.7 & 0.5 & 0.9 & 0.9 & 0.9 \\
\hline External grants & 3.7 & 3.5 & 0.5 & 0.2 & 1.1 & 0.9 & 2.2 & 2.3 & 3.0 & 3.1 & 2.2 & 2.2 \\
\hline Expenditure and net lending & 40.0 & 33.8 & 6.1 & 6.9 & 14.2 & 16.5 & 22.1 & 25.2 & 32.8 & 33.8 & 31.3 & 32.3 \\
\hline Current expenditure & 28.7 & 31.0 & 5.9 & 6.2 & 13.5 & 15.0 & 19.8 & 22.3 & 28.0 & 28.9 & 24.2 & 23.8 \\
\hline Primary current expenditure & 21.5 & 21.3 & 5.0 & 4.3 & 10.5 & 10.4 & 15.9 & 16.3 & 21.8 & 22.2 & 20.3 & 20.0 \\
\hline Wages and salaries & 7.9 & 7.1 & 1.6 & 1.4 & 3.2 & 3.1 & 4.8 & 4.8 & 6.4 & 6.6 & 5.9 & 5.9 \\
\hline Goods and services & 5.6 & 6.7 & 1.4 & 1.2 & 3.1 & 3.3 & 4.8 & 5.2 & 6.8 & 6.9 & 6.9 & 6.9 \\
\hline Transfers & 7.9 & 7.3 & 2.1 & 1.6 & 4.2 & 4.0 & 6.2 & 6.3 & 8.5 & 8.6 & 7.4 & 7.0 \\
\hline Social program of central government & 2.2 & 2.7 & 0.7 & 0.4 & 1.4 & 1.3 & 2.1 & 2.1 & 3.1 & 3.0 & 2.5 & 2.5 \\
\hline Transfers to public sector from central governmer & 2.5 & 1.9 & 0.6 & 0.5 & 1.2 & 1.2 & 1.7 & 1.8 & 2.3 & 2.4 & 2.1 & 1.8 \\
\hline Benefits and programs of Social Security Fund & 3.2 & 2.7 & 0.8 & 0.7 & 1.6 & 1.5 & 2.4 & 2.3 & 3.2 & 3.1 & 2.8 & 2.8 \\
\hline Other & 0.1 & 0.2 & 0.0 & 0.0 & 0.1 & 0.1 & 0.1 & 0.1 & 0.1 & 0.1 & 0.1 & 0.1 \\
\hline Interest payments due & 7.2 & 9.8 & 0.9 & 2.0 & 3.0 & 4.6 & 3.9 & 6.0 & 6.1 & 6.7 & 3.8 & 3.8 \\
\hline Foreign interest & 3.9 & 4.0 & 0.2 & 0.1 & 1.6 & 2.1 & 1.8 & 2.5 & 3.3 & 2.8 & 1.4 & 1.7 \\
\hline Domestic interest & 3.3 & 5.7 & 0.7 & 1.8 & 1.4 & 2.5 & 2.1 & 3.5 & 2.8 & 3.9 & 1.9 & 1.5 \\
\hline Capital expenditure & 2.1 & 5.7 & 0.9 & 0.7 & 2.2 & 2.6 & 4.3 & 4.9 & 6.8 & 7.4 & 7.3 & 8.5 \\
\hline Net lending & 9.1 & -2.9 & -0.8 & 0.0 & -1.7 & -1.1 & -2.6 & -2.4 & -3.0 & -3.1 & -0.2 & 0.0 \\
\hline Contingency & $\ldots$ & $\ldots$ & 0.0 & $\ldots$ & 0.2 & 0.0 & 0.6 & 0.3 & 1.1 & 0.5 & 0.0 & 0.0 \\
\hline Primary balance & 3.9 & 14.8 & 2.4 & 2.7 & 4.0 & 4.1 & 6.0 & 6.0 & 7.0 & 7.4 & 6.0 & 4.7 \\
\hline Overall balance, commitment & -3.4 & 5.0 & 1.5 & 0.7 & 1.0 & -0.5 & 2.1 & 0.0 & 0.9 & 0.7 & 2.1 & 0.8 \\
\hline Change in arrears & 0.4 & 0.9 & -0.2 & -0.1 & -0.5 & -0.3 & -0.7 & -0.6 & -0.9 & -0.9 & 0.0 & 0.0 \\
\hline External interest & 1.9 & 3.0 & 0.0 & 0.0 & 0.0 & 0.0 & 0.0 & 0.0 & 0.0 & 0.0 & 0.0 & 0.0 \\
\hline Budget & -1.6 & -2.1 & -0.2 & -0.1 & -0.5 & -0.3 & -0.7 & -0.6 & -0.9 & -0.9 & 0.0 & 0.0 \\
\hline Overall balance, cash basis (after grants) & -3.0 & 5.9 & 1.3 & 0.7 & 0.5 & -0.8 & 1.5 & -0.6 & 0.0 & -0.2 & 2.1 & 0.8 \\
\hline Financing & 3.0 & -5.9 & -1.3 & -0.7 & -0.5 & 0.8 & -1.5 & 0.6 & 0.0 & 0.2 & -2.1 & -0.8 \\
\hline Foreign financing & 2.7 & -1.6 & -1.2 & -0.4 & -2.6 & -1.2 & -2.9 & -0.9 & -1.8 & 0.4 & 1.3 & 3.1 \\
\hline Disbursements & 7.7 & 1.3 & 0.1 & 1.0 & 0.3 & 1.3 & 1.2 & 2.6 & 3.7 & 3.9 & 3.0 & 3.9 \\
\hline Project loans & 7.7 & 0.1 & 0.1 & 0.1 & 0.3 & 0.4 & 1.2 & 0.7 & 1.6 & 1.1 & 1.0 & 1.9 \\
\hline Program/budget support & 0.0 & 1.1 & 0.0 & 0.9 & 0.0 & 0.9 & 0.0 & 1.8 & 2.0 & 2.9 & 2.0 & 2.0 \\
\hline Scheduled amortization & -18.7 & -10.2 & -1.3 & -1.4 & -2.9 & -2.6 & -4.2 & -3.5 & -5.5 & -3.7 & -1.9 & -1.0 \\
\hline Change in amortization arrears & 13.7 & -11.3 & 0.0 & -17.6 & 0.0 & -17.4 & 0.0 & -17.4 & 0.0 & -17.4 & 0.0 & 0.0 \\
\hline Clearance of arrears & 0.0 & 18.1 & 0.0 & 17.6 & 0.0 & 17.4 & 0.0 & 17.4 & 0.0 & 17.4 & 0.0 & 0.0 \\
\hline Debt service relief & 0.0 & 0.5 & 0.0 & 0.0 & 0.1 & 0.1 & 0.1 & 0.1 & 0.1 & 0.1 & 0.1 & 0.1 \\
\hline Domestic financing & -0.8 & -6.6 & -0.5 & -1.2 & 1.1 & -0.8 & -0.1 & -2.7 & -4.7 & -6.5 & -6.2 & -5.7 \\
\hline Bank financing & -1.1 & -7.7 & -0.5 & -1.3 & 1.0 & -0.4 & -0.1 & -1.5 & -4.2 & -4.4 & -5.9 & -5.4 \\
\hline CBS & $\ldots$ & -3.5 & -0.2 & -1.6 & 2.1 & -1.6 & 1.8 & -1.6 & 0.4 & -1.6 & -3.1 & -2.8 \\
\hline CBS recapitalization & $\ldots$ & $\ldots$ & $\ldots$ & 0.0 & -1.8 & 0.6 & -1.8 & 1.1 & -1.8 & 1.7 & $\ldots$ & $\ldots$ \\
\hline Commercial banks & $\ldots$ & -4.2 & -0.3 & 0.3 & 0.7 & 0.7 & 0.0 & -1.1 & -2.8 & -4.5 & -2.8 & -2.6 \\
\hline Nonbank & 0.3 & 1.1 & -0.1 & 0.1 & 0.1 & 0.1 & 0.0 & -0.1 & -0.5 & -0.5 & -0.3 & -0.3 \\
\hline Privatization and long-term lease of fixed assets & 2.5 & 2.1 & 0.5 & 0.5 & 0.9 & 0.8 & 1.5 & 1.1 & 2.3 & 1.4 & 1.3 & 1.2 \\
\hline Statistical discrepancy & -1.4 & 0.2 & 0.0 & 0.5 & 0.0 & 0.5 & 0.0 & 0.5 & 0.0 & 0.5 & 0.0 & 0.0 \\
\hline Fiscal financing gap & 0.0 & 0.0 & -0.4 & 0.0 & 0.4 & 1.6 & -0.2 & 2.7 & 4.2 & 4.5 & 1.5 & 0.6 \\
\hline \multicolumn{13}{|l|}{ Memorandum items: } \\
\hline Nominal GDP & 8,710 & 10,726 & 11,057 & 11,206 & 11,057 & 11,206 & 11,057 & 11,206 & 11,057 & 11,206 & 12,091 & 13,059 \\
\hline Domestic debt (percent of revised GDP) & 53.9 & 36.0 & $\ldots$ & $\ldots$ & $\ldots$ & $\ldots$ & $\ldots$ & $\ldots$ & 37.4 & 28.8 & 23.6 & 19.0 \\
\hline
\end{tabular}

Sources: The Seychelles authorities and IMF staff estimates and projections.

' Includes the central government and the social security system. 
Table 4. Seychelles: Monetary Survey and Central Bank Accounts, 2008-10

\begin{tabular}{|c|c|c|c|c|c|c|c|c|c|c|c|}
\hline & \multirow[t]{3}{*}{2008} & \multirow{2}{*}{\multicolumn{2}{|c|}{2009}} & \multicolumn{8}{|c|}{2010} \\
\hline & & & & \multicolumn{2}{|c|}{ Mar. } & \multicolumn{2}{|c|}{ June $^{4}$} & \multicolumn{2}{|c|}{ Sep. ${ }^{4}$} & \multicolumn{2}{|c|}{ Dec. ${ }^{4}$} \\
\hline & & Prog. & Act. & Prog. & Act. & Prog. & Proj & Prog. & Proj & Prog. & Proj \\
\hline & \multicolumn{11}{|c|}{ (Seychelles rupees millions, unless otherwise indicated) } \\
\hline \multicolumn{12}{|l|}{ Monetary survey } \\
\hline Net foreign assets & 2,105 & 2,363 & 2,897 & 2,388 & 3,345 & 2,373 & 3,079 & 2,563 & 3,244 & 2,956 & 3,408 \\
\hline Central bank & 1,050 & 1,777 & 1,998 & 1,802 & 2,397 & 1,759 & 2,140 & 1,942 & 2,304 & 2,271 & 2,469 \\
\hline Deposit money banks & 1,055 & 586 & 899 & 586 & 947 & 614 & 939 & 621 & 941 & 684 & 939 \\
\hline Net domestic assets & 3,848 & 3,547 & 3,504 & 3,834 & 3,223 & 4,182 & 3,684 & 4,451 & 3,952 & 4,466 & 4,216 \\
\hline Domestic credit & 5,949 & 4,410 & 4,796 & 4,518 & 4,811 & 4,948 & 5,028 & 5,069 & 5,018 & 4,878 & 4,866 \\
\hline Net claims on the government & 3,082 & 1,722 & 2,255 & 1,707 & 2,115 & 1,995 & 2,216 & 1,934 & 2,082 & 1,457 & 1,761 \\
\hline Credit to the economy & 2,867 & 2,688 & 2,540 & 2,811 & 2,695 & 2,954 & 2,813 & 3,135 & 2,936 & 3,421 & 3,105 \\
\hline Other items, net & $-2,102$ & -863 & $-1,292$ & -684 & $-1,587$ & -766 & $-1,344$ & -618 & $-1,066$ & -413 & -650 \\
\hline Broad money & 5,952 & 5,910 & 6,401 & 6,222 & 6,568 & 6,555 & 6,763 & 7,015 & 7,197 & 7,421 & 7,623 \\
\hline Currency in circulation & 478 & 503 & 500 & 509 & 497 & 528 & 519 & 558 & 549 & 587 & 577 \\
\hline Foreign currency deposits & 1,913 & 1,514 & 1,610 & 1,600 & 1,682 & 1,687 & 1,655 & 1,808 & 1,762 & 1,914 & 1,867 \\
\hline Local currency deposits & 3,562 & 3,893 & 4,291 & 4,113 & 4,388 & 4,339 & 4,589 & 4,648 & 4,886 & 4,921 & 5,179 \\
\hline \multicolumn{12}{|l|}{ Central bank } \\
\hline Net foreign assets & 1,050 & 1,777 & 1,998 & 1,802 & 2,397 & 1,759 & 2,140 & 1,942 & 2,304 & 2,271 & 2,469 \\
\hline Foreign assets & 1,207 & 1,982 & 2,207 & 2,044 & 2,648 & 2,040 & 2,468 & 2,264 & 2,675 & 2,627 & 2,908 \\
\hline Of which: official reserves & 843 & 1,689 & 1,907 & 1,740 & 2,470 & 1,735 & 2,267 & 1,954 & 2,473 & 2,302 & 2,704 \\
\hline Foreign liabilities & 157 & 205 & 210 & 242 & 250 & 281 & 329 & 322 & 372 & 355 & 439 \\
\hline Net domestic assets & 70 & -297 & -702 & -266 & -973 & -168 & -548 & -268 & -630 & -518 & -716 \\
\hline Domestic credit & 851 & -260 & -657 & -279 & -779 & 27 & -295 & -48 & -302 & -274 & -311 \\
\hline Government (net) $^{1}$ & 1,099 & 60 & 724 & 55 & 547 & 284 & 609 & 264 & 672 & 105 & 735 \\
\hline Commercial banks & -22 & -198 & $-1,266$ & -212 & $-1,202$ & -135 & -781 & -190 & -851 & -257 & -922 \\
\hline Other (parastatals) & -226 & -122 & -114 & -122 & -123 & -122 & -123 & -122 & -123 & -122 & -123 \\
\hline Other items, net & -780 & -37 & -45 & 13 & -195 & -196 & -254 & -221 & -328 & -245 & -405 \\
\hline Reserve money & 1,120 & 1,480 & 1,296 & 1,536 & 1,424 & 1,591 & 1,591 & 1,674 & 1,674 & 1,753 & 1,753 \\
\hline Currency in circulation & 478 & 503 & 500 & 509 & 497 & 528 & 519 & 558 & 549 & 587 & 577 \\
\hline Commercial bank reserves (includes cash in vault) ${ }^{2}$ & 642 & 977 & 796 & 1,028 & 927 & 1,063 & 1,072 & 1,116 & 1,125 & 1,166 & 1,176 \\
\hline o.w. required reserves in foreign currency & $\ldots$ & 150 & 170 & 161 & 176 & 172 & 188 & 183 & 200 & 195 & 212 \\
\hline required reserves in domestic currency & 745 & $\ldots$ & 441 & $\ldots$ & 453 & $\ldots$ & 473 & $\ldots$ & 502 & $\ldots$ & 532 \\
\hline \multicolumn{12}{|l|}{ Memorandum items: } \\
\hline Gross international reserves (US\$ millions) ${ }^{3}$ & 51 & 153 & 169 & 158 & 210 & 156 & 192 & 174 & 207 & 202 & 224 \\
\hline Foreign Currency Deposits (US\$ millions) & 115 & 137 & 143 & 145 & 143 & 152 & 140 & 161 & 147 & 168 & 154 \\
\hline Broad money growth (12-month percent change) & 27.2 & -0.7 & 7.5 & 15.0 & 21.4 & 29.4 & 33.5 & 29.6 & 33.0 & 25.6 & 19.1 \\
\hline Credit to the economy (12-month percent change) & 48.6 & -12.1 & -11.4 & -2.7 & 2.9 & 10.1 & 14.3 & 19.3 & 19.1 & 27.3 & 22.2 \\
\hline Reserve money (12-month percent change) & 0.6 & 32.2 & 15.7 & 46.2 & 35.5 & 42.1 & 42.2 & 43.2 & 43.1 & 18.4 & 35.3 \\
\hline Money multiplier (broad money/reserve money) & 5.3 & 4.0 & 4.9 & 4.1 & 4.6 & 4.1 & 4.3 & 4.2 & 4.3 & 4.2 & 4.3 \\
\hline Velocity (GDP/broad money; end of period) & 1.5 & 1.8 & 1.7 & 1.5 & 1.5 & 1.5 & 1.5 & 1.5 & 1.5 & 1.5 & 1.5 \\
\hline Change in Net Credit to Government (change in quarter) & -158 & -386 & 148 & -15 & -140 & 287 & 100 & -60 & -133 & -478 & -321 \\
\hline
\end{tabular}

Sources: Central Bank of Seychelles and IMF staff estimates and projections.

${ }^{1}$ The difference compared to the program was due to unplanned large repayment in Q4 of Tbills to the private sector out of government deposits at the CBS.

${ }^{2}$ Reserve requirements were lowered from $13 \%$ to $10 \%$ in Q4 2009.

${ }^{3}$ Reflects an increase in SDR holdings following SDR allocation of SDR 7.9 million in Q3 2009.

${ }^{4}$ Assumes recapitalization of the CBS by SR 188 million in three equal quarterly installments. 
Table 5. Seychelles: Financial Soundness Indicators for the Banking Sector, 2007-10 ${ }^{1}$ (Percent, end of period)

\begin{tabular}{|c|c|c|c|c|c|c|c|c|c|c|c|c|c|}
\hline & \multicolumn{4}{|c|}{2007} & \multicolumn{4}{|c|}{2008} & \multicolumn{4}{|c|}{2009} & \multirow{2}{*}{$\frac{2010}{\mathrm{Q} 1}$} \\
\hline & Q1 & Q2 & Q3 & Q4 & Q1 & Q2 & Q3 & Q4 & Q1 & Q2 & Q3 & Q4 & \\
\hline \multicolumn{14}{|l|}{ Capital adequacy ${ }^{2}$} \\
\hline Regulatory capital to risk weighted assets & 20.4 & 21.0 & 18.2 & 18.2 & 18.4 & 17.6 & 17.9 & 19.4 & 13.6 & 17.8 & 22.0 & 21.7 & 21.4 \\
\hline Regulatory tier 1 capital to risk weighted assets & 14.8 & 16.1 & 14.8 & 12.9 & 12.9 & 14.0 & 13.4 & 10.4 & 12.9 & 17.1 & 21.3 & 21.0 & 20.7 \\
\hline Capital to assets (net worth) & 6.2 & 6.5 & 6.4 & 6.4 & 6.9 & 6.6 & 7.0 & 8.4 & 9.6 & 8.7 & 9.7 & 9.9 & 10.3 \\
\hline Net tangible capitalization ${ }^{3}$ & 6.2 & 6.6 & 6.5 & 6.5 & 7.0 & 6.6 & 7.1 & 8.4 & 9.6 & 8.7 & 9.8 & 9.9 & 10.4 \\
\hline \multicolumn{14}{|l|}{ Asset quality } \\
\hline Foreign exchange loans to total loans & 24.1 & 25.1 & 28.7 & 30.8 & 32.7 & 32.9 & 31.6 & 50.8 & 50.5 & 43.0 & 41.1 & 37.2 & 34.3 \\
\hline Nonperforming loans to gross loans & 3.7 & 2.9 & 2.5 & 2.3 & 2.0 & 2.1 & 2.0 & 2.0 & 2.3 & 4.3 & 4.2 & 3.8 & 4.1 \\
\hline Provisions as percentage of nonperforming loans & 38.2 & 44.1 & 45.6 & 45.9 & 51.2 & 50.3 & 59.6 & 58.3 & 56.8 & 35.2 & 32.6 & 33.8 & 41.7 \\
\hline \multicolumn{14}{|l|}{ Earnings and profitability } \\
\hline Return on assets (annualized) & 3.9 & 3.4 & 5.0 & 6.6 & 4.3 & 3.4 & 2.8 & 16.1 & 5.3 & 3.0 & -0.2 & 3.2 & 3.4 \\
\hline Return on equity (annualized) & 59.1 & 50.4 & 68.3 & 93.0 & 59.5 & 47.9 & 39.3 & 199.4 & 55.1 & 35.4 & -2.8 & 32.4 & 33.6 \\
\hline Interest margin to gross income & 60.0 & 66.1 & 57.9 & 46.0 & 58.7 & 62.8 & 58.3 & 24.2 & 62.1 & 83.7 & 105.5 & 68.6 & 56.5 \\
\hline Noninterest expense to gross income & 35.5 & 39.7 & 33.3 & 32.3 & 33.2 & 43.5 & 49.4 & 17.8 & 36.4 & 48.7 & 116.5 & 49.1 & 44.0 \\
\hline Net interest margin (annualized) ${ }^{4}$ & 3.6 & 3.7 & 4.3 & 4.5 & 3.8 & 3.8 & 3.1 & 4.8 & 5.1 & 4.8 & 4.2 & 4.0 & 3.2 \\
\hline Net noninterest margin (annualized) $)^{5}$ & 0.3 & -0.3 & 0.6 & 2.1 & 0.5 & -0.4 & -0.4 & 11.4 & 0.1 & -1.9 & -4.9 & -1.0 & 0.0 \\
\hline Expense to income & 48.5 & 53.5 & 44.8 & 42.2 & 46.6 & 55.9 & 62.5 & 25.5 & 52.3 & 62.2 & 111.2 & 58.0 & 53.0 \\
\hline Interest expense to gross income & 25.2 & 29.5 & 20.8 & 17.2 & 25.0 & 28.0 & 35.0 & 10.2 & 33.2 & 35.8 & 47.1 & 21.2 & 19.0 \\
\hline \multicolumn{14}{|l|}{ Liquidity } \\
\hline Core liquid assets to total assets ${ }^{6}$ & 19.2 & 18.6 & 11.4 & 10.0 & 30.8 & 34.6 & 36.4 & 37.8 & 36.0 & 35.0 & 37.7 & 43.2 & 45.2 \\
\hline Broad liquid assets to total assets ${ }^{7}$ & 54.8 & 55.8 & 44.3 & 39.4 & 59.2 & 60.9 & 60.0 & 55.7 & 50.8 & 53.0 & 57.6 & 58.4 & 61.0 \\
\hline Liquid assets (broad) to short term liabilities & 57.3 & 57.5 & 46.7 & 40.8 & 59.3 & 61.6 & 87.1 & 56.5 & 53.2 & 56.2 & 62.1 & 62.7 & 65.0 \\
\hline Liquid assets (broad) to total liabilities & 58.7 & 60.1 & 47.8 & 42.3 & 63.9 & 65.5 & 64.8 & 61.2 & 56.2 & 58.0 & 63.8 & 64.7 & 68.0 \\
\hline Liquid assets to deposit liabilities & 64.0 & 64.5 & 52.5 & 46.0 & 68.3 & 72.4 & 71.7 & 66.4 & 63.1 & 63.8 & 71.4 & 69.4 & 72.4 \\
\hline \multicolumn{14}{|l|}{ Foreign exchange exposure } \\
\hline Net open foreign exchange position to capital & 2.6 & 14.1 & 28.3 & 28.5 & 24.9 & 31.3 & 21.3 & 58.9 & 44.4 & 32.4 & 21.9 & 33.1 & 20.9 \\
\hline
\end{tabular}

${ }^{1}$ Excluding purely offshore banks.

2 Under new standards of provisioning and capital adequacy.

${ }^{3}$ Defined as: equity capital/(assets-interest in suspense-provisions)

${ }^{4}$ Defined as: (Interest income - interest expense)/average assets.

${ }^{5}$ Defined as: (Noninterest income - noninterest expense)/average assets

${ }^{6}$ Core liquid assets include cash, balances with CBS and deposits $w$ ith other banks

${ }^{7}$ Broad liquid assets include core liquid assets plus investments in government securities. 


\section{Table 6. Seychelles: Fund Disbursements and Timing of Reviews Under the Proposed Three-Year EFF, 2009-12}

\begin{tabular}{|c|c|c|c|c|}
\hline \multirow{2}{*}{$\begin{array}{l}\text { Program } \\
\text { Review }\end{array}$} & \multirow[b]{2}{*}{ Date of availability } & \multirow[b]{2}{*}{ Conditions } & \multicolumn{2}{|c|}{ Amount } \\
\hline & & & (millio & (percent of quota) \\
\hline & December 22, 2009 & Board approval of the EFF arrangement. & 0.88 & 10 \\
\hline & March 15, 2010 & $\begin{array}{l}\text { Compliance with end-December } 2009 \\
\text { quantitative performance criteria and } \\
\text { completion of a financing assurances review (if } \\
\text { needed) }\end{array}$ & 2.20 & 25 \\
\hline \multirow[t]{2}{*}{ First } & June 15, 2010 & $\begin{array}{l}\text { Completion of first program review and } \\
\text { financing assurances review (if needed) and } \\
\text { compliance with end-March } 2010 \text { quantitative } \\
\text { performance criteria. }\end{array}$ & 2.20 & 25 \\
\hline & September 15, 2010 & $\begin{array}{l}\text { Compliance with end-June } 2010 \text { quantitative } \\
\text { performance criteria and completion of a } \\
\text { financing assurances review (if needed) }\end{array}$ & 2.20 & 25 \\
\hline \multirow[t]{2}{*}{ Second } & December 15, 2010 & $\begin{array}{l}\text { Completion of second program review and } \\
\text { financing assurances review (if needed) and } \\
\text { compliance with end-September } 2010 \\
\text { quantitative performance criteria. }\end{array}$ & 1.76 & 20 \\
\hline & March 15, 2011 & $\begin{array}{l}\text { Compliance with end-December } 2010 \\
\text { quantitative performance criteria and } \\
\text { completion of a financing assurances review (if } \\
\text { needed). }\end{array}$ & 1.76 & 20 \\
\hline \multirow[t]{2}{*}{ Third } & June 15, 2011 & $\begin{array}{l}\text { Completion of third program review and a } \\
\text { financing assurances review (if needed) and } \\
\text { compliance with end-March } 2011 \text { quantitative } \\
\text { performance criteria) }\end{array}$ & 1.76 & 20 \\
\hline & September 15, 2011 & $\begin{array}{l}\text { Compliance with end-June } 2011 \text { quantitative } \\
\text { performance criteria and completion of a } \\
\text { financing assurances review (if needed) }\end{array}$ & 1.76 & 20 \\
\hline \multirow[t]{2}{*}{ Fourth } & December 15, 2011 & $\begin{array}{l}\text { Completion of fourth program review and a } \\
\text { financing assurances review (if needed) and } \\
\text { compliance with end-September } 2011 \\
\text { quantitative performance criteria) }\end{array}$ & 1.32 & 15 \\
\hline & March 15, 2012 & $\begin{array}{l}\text { Compliance with end-December } 2011 \\
\text { quantitative performance criteria and } \\
\text { completion of a financing assurances review (if } \\
\text { needed) }\end{array}$ & 1.32 & 15 \\
\hline \multirow[t]{2}{*}{ Fifth } & June 15, 2012 & $\begin{array}{l}\text { Completion of fifth program review and a } \\
\text { financing assurances review (if needed) and } \\
\text { compliance with end-March } 2012 \text { quantitative } \\
\text { performance criteria) }\end{array}$ & 1.32 & 15 \\
\hline & September 15, 2012 & $\begin{array}{l}\text { Compliance with end-June } 2012 \text { quantitative } \\
\text { performance criteria and completion of a } \\
\text { financing assurances review (if needed) }\end{array}$ & 1.32 & 15 \\
\hline Total & & & 19.8 & 225 \\
\hline
\end{tabular}


Table 7. Seychelles: Structural Benchmarks, 2009-10

\begin{tabular}{|c|c|c|c|}
\hline Measure & Target date & Macroeconomic rationale & Status \\
\hline $\begin{array}{l}\text { Introduce a treasury single } \\
\text { account. }\end{array}$ & $\begin{array}{l}\text { End- } \\
\text { September } 2009\end{array}$ & $\begin{array}{l}\text { To strengthen public } \\
\text { finances, key to sustainability } \\
\text { efforts. }\end{array}$ & $\begin{array}{l}\text { Met. The TSA is } \\
\text { leveraging progress on } \\
\text { PFM. }\end{array}$ \\
\hline $\begin{array}{l}\text { Adopt foreign reserves } \\
\text { management investment } \\
\text { guidelines. }\end{array}$ & $\begin{array}{l}\text { End- } \\
\text { September } 2009\end{array}$ & $\begin{array}{l}\text { To ensure management of } \\
\text { foreign reserves in line with } \\
\text { best international practice. }\end{array}$ & $\begin{array}{l}\text { Met. The CBS Board } \\
\text { adopted guidelines on } \\
\text { September 25, } 2009 .\end{array}$ \\
\hline $\begin{array}{l}\text { Adopt Public Enterprise } \\
\text { Monitoring and Control act. }\end{array}$ & $\begin{array}{l}\text { End- } \\
\text { September } 2009\end{array}$ & $\begin{array}{l}\text { To improve oversight over } \\
\text { public enterprises and reduce } \\
\text { quasi-fiscal risks. }\end{array}$ & $\begin{array}{l}\text { Met. Parliamentary } \\
\text { approval secured on } \\
\text { September } 28,2009 \text {. }\end{array}$ \\
\hline $\begin{array}{l}\text { Complete CBS procedures } \\
\text { manual (MEFP, } ₫ 46) \text {. }\end{array}$ & $\begin{array}{l}\text { End- } \\
\text { December } 2009\end{array}$ & $\begin{array}{l}\text { To support improved } \\
\text { efficiency and transparency } \\
\text { in monetary policy. }\end{array}$ & $\begin{array}{l}\text { Met. The manual was } \\
\text { approved on } \\
\text { December } 29,2009 \text {. }\end{array}$ \\
\hline 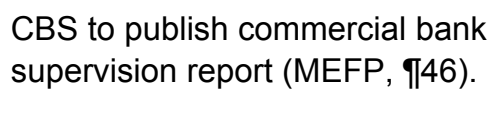 & $\begin{array}{l}\text { End- } \\
\text { December } 2009\end{array}$ & $\begin{array}{l}\text { To promote competition and } \\
\text { transparency in the banking } \\
\text { system. }\end{array}$ & $\begin{array}{l}\text { Met. Published on the } \\
\text { CBS website on } \\
\text { December 29, } 2009\end{array}$ \\
\hline $\begin{array}{l}\text { Amend the Business Tax Act in } \\
\text { line with the tax reform strategy } \\
\text { (MEFP, } \mid 22 \text { ). }\end{array}$ & $\begin{array}{l}\text { End- } \\
\text { December } 2009\end{array}$ & $\begin{array}{l}\text { To broaden the tax base, } \\
\text { modernize tax policy, and } \\
\text { remove distortions. }\end{array}$ & Met. \\
\hline $\begin{array}{l}\text { Cabinet approval of customs } \\
\text { reform strategy and } \\
\text { implementation plan (MEFP, } \\
\text { \23). }\end{array}$ & End-March 2010 & $\begin{array}{l}\text { To transform customs into a } \\
\text { modern and efficient entity. }\end{array}$ & $\begin{array}{l}\text { Met. The approval was } \\
\text { secured on } \\
\text { March 17, } 2010 .\end{array}$ \\
\hline $\begin{array}{l}\text { Publish general government } \\
\text { fiscal statistics (MEFP, } \mid 29 \text { ). }\end{array}$ & End-April 2010 & $\begin{array}{l}\text { To enhance transparency } \\
\text { and coverage of public } \\
\text { finance. }\end{array}$ & $\begin{array}{l}\text { Met. Fiscal statistics } \\
\text { were published on } \\
\text { April 23, } 2010\end{array}$ \\
\hline $\begin{array}{l}\text { Submit to National Assembly a } \\
\text { new customs management act } \\
\text { (MEFP, \23). }\end{array}$ & End-June 2010 & $\begin{array}{l}\text { To institutionalize best } \\
\text { international practice and a } \\
\text { better business climate. }\end{array}$ & $\begin{array}{l}\text { Moved to end- } \\
\text { September 2010, to } \\
\text { address regional } \\
\text { harmonization issues. }\end{array}$ \\
\hline $\begin{array}{l}\text { Introduce Personal Income Tax } \\
\text { (MEFP, \22). }\end{array}$ & July 1, 2010 & $\begin{array}{l}\text { To broader the tax base and } \\
\text { provide for more equitable } \\
\text { taxation. }\end{array}$ & \\
\hline $\begin{array}{l}\text { Introduce budget submissions } \\
\text { protocols and procedures } \\
\text { (MEFP, 『28). }\end{array}$ & End-July 2010 & $\begin{array}{l}\text { To strengthen budget } \\
\text { preparation. }\end{array}$ & \\
\hline $\begin{array}{l}\text { Adopt a new chart of account for } \\
\text { the } 2011 \text { budget (MEFP, } \uparrow 29 \text { ). }\end{array}$ & $\begin{array}{l}\text { End- } \\
\text { November } 2010\end{array}$ & $\begin{array}{l}\text { To ensure proper } \\
\text { classification and increase } \\
\text { efficiency of the budget as a } \\
\text { policy tool. }\end{array}$ & \\
\hline $\begin{array}{l}\text { Submit to National Assembly a } \\
\text { bill creating a national clearing } \\
\text { house and settlement system } \\
\text { (MEFP, } \llbracket 44 \text { ). }\end{array}$ & $\begin{array}{l}\text { End- } \\
\text { December } 2010\end{array}$ & $\begin{array}{l}\text { To introduce necessary } \\
\text { financial infrastructure for } \\
\text { improved efficiency and } \\
\text { reduced risks. }\end{array}$ & \\
\hline
\end{tabular}


Figure 1. Seychelles: Macroeconomic Developments
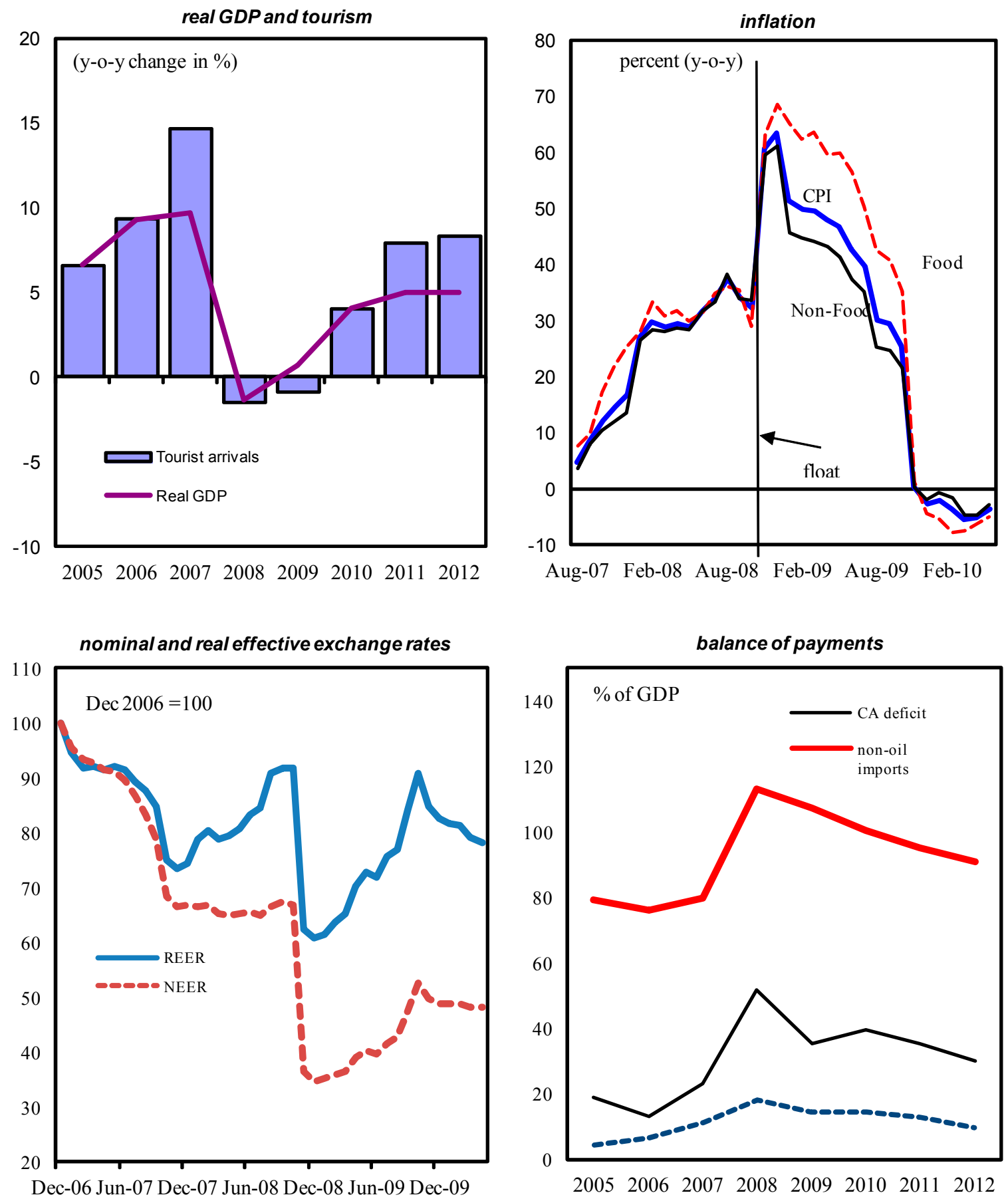

Source: Seychelles authorities and Fund staff estimates. 
Figure 2. Seychelles: Fiscal Sector
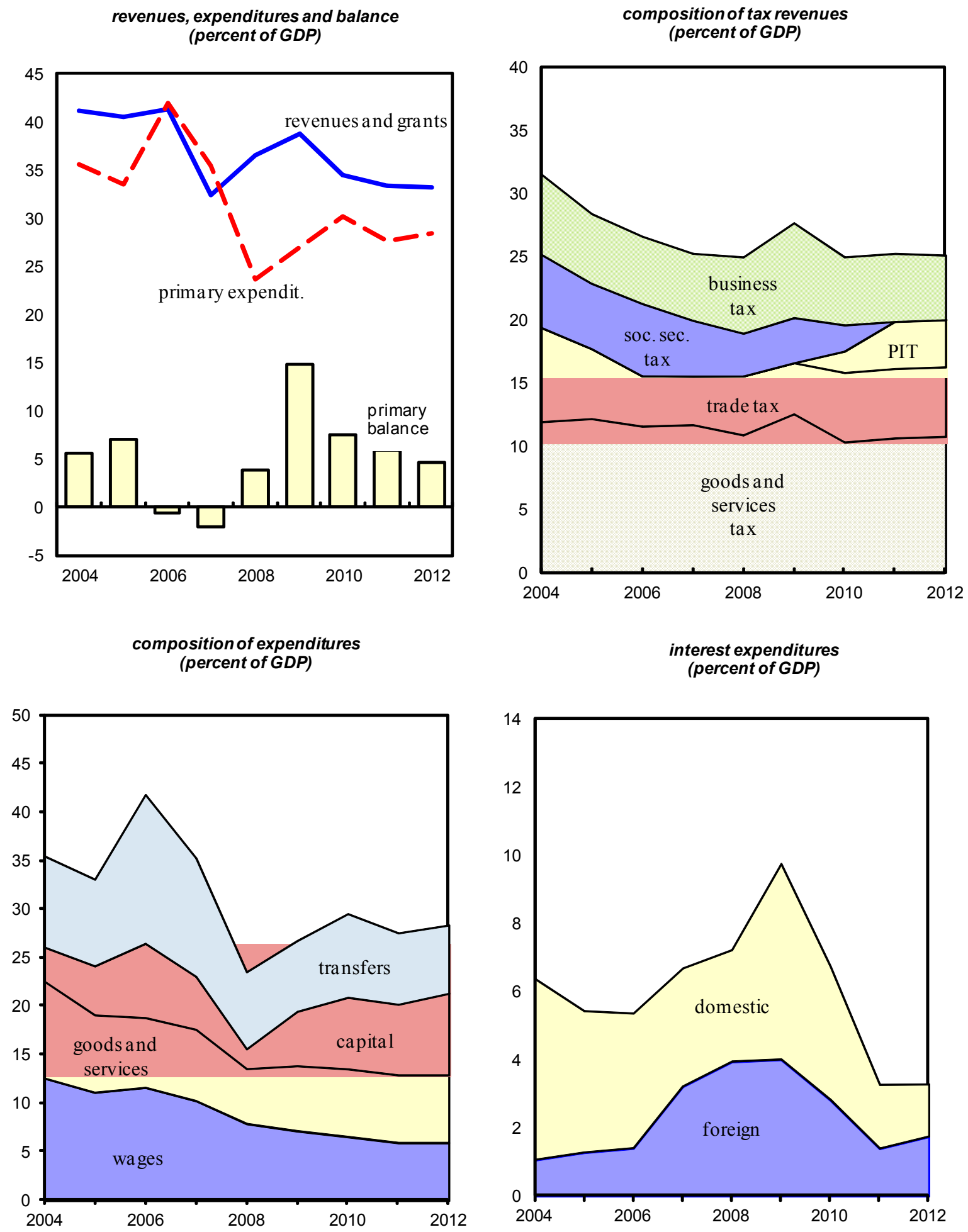

Source: Seychelles authorities and Fund staff estimates. 


\section{APPENDIX I \\ SEYCHELLES: LETTER OF INTENT}

June 1, 2010

Mr. Dominique Strauss-Kahn

Managing Director

International Monetary Fund

Washington, D.C. 20431

Dear Mr. Strauss-Kahn:

1. The attached Supplementary Memorandum of Economic and Financial Policies for 2010-12 (MEFP) describes Seychelles' performance through end-April 2010 and updates macroeconomic and structural policies for the remainder of 2010, as well as our priorities through 2012. This MEFP complements and updates the MEFP of December 3, 2009 which set out the objectives of our three-year program supported by an arrangement under the Extended Fund Facility (EFF).

2. We request completion of the first review under our EFF arrangement and the release of the third tranche of SDR 2.2 million (25 percent of quota) there under. All the quantitative performance criteria (PCs) at end-March 2009 were met and the two structural benchmarks for end-March and end-April 2010 respectively were observed. We also request the modification of the quantitative performance criterion on the fiscal primary balance.

3. We believe that the economic and financial policies set forth in the MEFP are sufficient to ensure that the objectives of the program will be met. We stand ready to take any further measures that may prove necessary to meet our objectives. We will consult with the Fund on the adoption of these measures, and in advance of revisions to the policies contained in the MEFP, in accordance with the Fund's policies on such consultations.

4. The completion of the second review under the EFF will be based on meeting the quantitative PCs at end-September 2010 and is scheduled to be completed by endDecember 2010. Quarterly financing assurance reviews will continue as long as public debt arrears remain outstanding. 
5. In line with our commitment to transparency, we request that the IMF publish this letter, the MEFP, and the Technical Memorandum of Understanding. We will simultaneously publish these documents in Seychelles.

Sincerely yours,

$$
\text { /s/ }
$$

James Alix Michel

President

Republic of Seychelles

Attachments: SMEFP and TMU 


\section{AtTAChment I}

\section{SEYCHELles: SuPPlementary MEMORANDUM OF ECONOMIC, FinANCIAL AND STRUCTURAL REFORM POLICIES FOR 2010-12}

This document:

\section{INTRODUCTION}

- Reviews macroeconomic and financial performance under our Extended Fund Facility (EFF) arrangement through end-April 2010.

- Updates the MEFP of December 3, 2009, detailing our policy commitments through the remainder of 2010 to support completion of the second EFF review, as well as for the three year period 2010-2012 under the EFF arrangement.

\section{MACROECONOMIC AND FINANCIAL CONTEXT}

1. Macroeconomic trends continue to evolve favorably, with recent developments generally exceeding expectations. Economic activity was flat in 2009, rather than the anticipated contraction, consistent with early signs of recovery in the second half of the year. This positive momentum has continued into the first quarter of 2010 , underpinned by an increase in tourist arrivals. Tourism activity has been maintained through deep discounting that has contributed to a significant decline in the terms of trade. Since December 2009 the nominal effective exchange rate has stabilized, with the rupee depreciating against the US dollar and appreciating against the Euro in similar proportions. Inflationary pressures remain well-contained, with the year-on-year inflation rate falling to -5.1 percent in April. T-Bill rates have declined to around 3 percent (91 day), consistent with tight fiscal policy and a measured easing in monetary conditions. However, credit growth has been slow to pick up despite adequate liquidity conditions, consistent with the slight recovery in domestic activity to date and with commercial lending rates adjusting only gradually to the financial sector's lower cost of borrowing. The reconstitution of gross international reserves has progressed strongly to \$US190 million (2 months of imports) at end April 2010.

\section{Commercial banks remain well capitalized and there are no signs of}

deterioration in asset quality in the financial sector. Based on first quarter 2010 data, the capitalization level of banks stood at 21.5 percent measured according to the Basel 1 capitalization standard and 10.4 percent on a net tangible capitalization basis. Total assets of the banking sector increased by 1 percent from December 2009 to March 2010. Relative to total assets, total credit remained at a low level, growing by a modest 3 percent. Notwithstanding the economic slowdown, nonperforming loans as a percentage of total loans have remained at 4 percent, indicating that credit remains of a high quality. 
3. There was no significant change in interest rates over the quarter. The average weighted interest rate on savings deposits has remained stable at around 1.85 percent, which is a reflection of the high liquidity in the banking system. On the lending side, despite the reduction in prime lending rate by 1.9 percentage points to 10.9 percent in March, the average lending rate fell by only 43 basis points. The margin between the average lending and savings rates stood at 11.2 percent, highlighting the need for strengthened competition in our banking sector.

4. The outlook is for economic recovery to gain traction from $\mathbf{2 0 1 0}$ as activity responds to our first generation of reforms. We are projecting that real GDP will grow by 4 percent in 2010, building on the momentum in the second half of 2009 , and driven by a projected 8 percent rise in tourism arrivals. Deflationary pressures are expected to wane as economic activity picks up and the pass through of the rupee appreciation experienced in 2009 abates. Risks to the improved economic outlook include the potential impact on tourism and related investment of economic developments in Europe and any further disruption to air services arising from volcanic activity in Iceland. Piracy in the Indian Ocean has had a negative impact on both our fishing and tourism sectors, with any further escalation presenting both economic and fiscal risks.

\section{Program Implementation and 2010 Program Update}

\section{The major objectives of our EFF-supported program are to:}

a. consolidate macroeconomic stability;

b. complete debt restructuring and achieve external sustainability; and

c. improve economic efficiency and durably raise growth through the implementation of a second generation of structural reforms.

6. Our ambitious structural reform agenda aims to transform the economy by institutionalizing higher governance standards, rationalizing the public sector and improving the business environment. As detailed below, we are making good progress against the three structural reform pillars established under our EFF arrangement - public enterprise restructuring, tax reform, and strengthened public financial management. Our EFF arrangement also provides the macroeconomic framework for working with other development partners (such as the World Bank, African Development Bank and European Union) on enhancing the role of the private sector in the economy.

7. The government has continued to exceed its fiscal targets and is determined to maintain its strong track record in this regard. The primary fiscal surplus in 2009 was 14.9 percent of GDP, representing a fiscal contraction of some 11 percentage points of GDP compared to 2008. The primary surplus for the first quarter was 2.7 percent of GDP, exceeding the target established at the commencement of our EFF arrangement by 
0.3 percentage points. This result was driven by both lower expenditures and higher revenue collection, in part due to stronger than anticipated economic activity in 2009 and the recent depreciation of the rupee (boosting GST on imports).

8. Price stability remains the primary objective of monetary policy. Our success in containing inflation over the past year, and the significant appreciation of the rupee, allowed for a loosening of monetary policy from the third quarter of 2009, which has arrested the decline in prices in April 2010. While a buildup of liquidity - due in part to faster-thanexpected repayment of domestic debt by the government - has made monetary policy more challenging, we have succeeded in meeting both our reserve money and net international reserves targets with margins. We remain committed to the float of the rupee and to completing the move to indirect monetary instruments as conditions allow. The CBS continues to closely monitor credit and price developments and stands ready to tighten monetary policy should inflationary pressures emerge.

9. We have made significant progress in normalizing relations with external creditors. Reflecting this, the external debt stock declined from 92 percent of GDP in 2009 to 54 percent of GDP in March 2010. Fitch Ratings assigned a single-B rating with a positive outlook to external debt, and declared that it considered the Seychelles default to be cured.

10. All quantitative PCs at end-March were met with margins (Table 1) and we have implemented all structural benchmarks scheduled for completion prior to the first EFF review (Table 2). Table 4 provides an update of our broader economic reform program, both adding to and updating the timing of measures detailed in our previous MEFP.

\section{Fiscal Policy}

11. The overarching goal of fiscal policy remains to put public finances on a sustainable path, while creating the fiscal space to raise public investment and support our targeted social safety net. At the onset of the program, primary fiscal surpluses were targeted at 13.4 of GDP in 2009, 7 percent of GDP in 2010, declining to about $4 \frac{1}{2}$ percent by 2012 . We have exceeded the 2009 target and plan to also exceed the program target for 2010, which will accelerate progress toward fiscal sustainability, assuming full external debt restructuring, and allow us to further reduce domestic public debt, releasing resources to support private sector development.

\section{BUDGET}

12. Based on the outcome of the first four months, we now project higher government revenue by the equivalent of 1 percent of GDP, half of which will be saved. Higher projected revenue comes from a combination of higher dividends from several public enterprises and revenue over performance in the first four months of 2010. Additional 
current spending includes the cost of termination benefits associated with extending our program of outsourcing noncore functions beyond ministries and departments to all budgetdependent agencies, the higher-than-budgeted cost of the new wage grid and scheme of service for teachers and health professionals (with a view to arresting the ongoing exodus to the private sector), as well as urgent but unforeseen spending on goods and services. The fiscal margin freed by the accelerated repayment to the government of loans to public entities and the partial release of the capital expenditure contingency agreed at the commencement of our EFF arrangement will fund additional infrastructure as well as the increased cost of some investment projects. Looking ahead, SR 35 million will be released from the contingency provided the primary balance through June 2010 remains on track; and the remaining 25 million will be released provided the primary balance through endSeptember 2010 remains on track.

\section{TAX REFORM}

\section{We launched a comprehensive reform of the tax system with the 2010 budget.}

Our objective is to have a simple, fair and equitable system, which will promote growth, improve self compliance, and level the playing field for investors. Our tax reform comprises three pillars:

a. The first stage was the introduction of a revised Business Tax Act from January 1, 2010. The business tax reform broadened the tax base and provided for a step-wise reduction in rates to promote competitiveness and achieve harmonization across sectors. As a first step, the maximum rate has been revised downward by 7 percentage points, to 33 percent. The tax-free threshold was abolished for companies and reduced for sole traders and partnerships.

b. The second stage will be the introduction of a withholding-based personal income tax (PIT) from 1 July, 2010 on wages to replace social security contributions, expanding the labor income tax base to resident expatriates and eliminating existing sectoral concessions. Government policy is to broaden the application of the PIT to other sources of domestic-sourced income (e.g., dividends and interest on savings) once the PIT becomes established and the new system has been assessed. PIT rates will be harmonized at 15 percent, for all categories of workers, effective January 1, 2011. The PIT legislation has been approved by Cabinet and will be introduced in the National Assembly in June 2010.

c. The third pillar of the Government's tax reform strategy is the introduction of a Value Added Tax (VAT) from January 1, 2012. The VAT will replace the current multiple rate GST, broadening the indirect tax base and improving both the efficiency of the indirect tax system and external competitiveness. Important steps in this direction were taken in the 2010 Budget, including expanding the GST base to a broader range of services and the reduction of cascading effects through the option of 
levying GST either on imports or sales. The concessional 10 percent GST rate that currently applies in the tourism sector will be raised to 12 percent in November 2010, prior to alignment with the general GST rate of 15 percent by November 2011 in preparation for the introduction of the VAT. The design of and preparation for the VAT will be supported by technical assistance financed by the EU ( $€ 1$ million has been set aside for this purpose in 2010).

14. The reforms are expected to be broadly revenue neutral over the medium-term and will be accompanied by efforts to modernize and reinforce revenue administration. With a view to establishing a level playing field for all businesses by the time of the VAT introduction, we have also maintained our policy of not providing new tax incentives and exemptions and made progress on our goal to phase out existing ones.

\section{Several steps have been taken to improve SRC governance, including Cabinet} approval of a new organizational structure in December 2009 and the passing of the Revenue Administration Act and the Business Number Act. We plan now to accelerate the restructuring of SRC operations through the setting up of an audit group focused on large taxpayers. The new structure is expected to be fully operational by July 2010 . On customs, we are starting to implement the customs reform strategy and implementation plan that the Cabinet approved in March. We also plan to submit to National Assembly a new Customs Management Act by end- September 2010.

\section{PUBLIC FinANCIAL MANAGEMENT AND EXPENDITURE REFORM}

16. We are making considerable progress towards strengthening public financial management, in line with recommendations from the IMF's Fiscal Affairs

Department. The introduction of a Treasury Single Account (TSA) in late 2009 represented a major step forward in our capacity to both monitor and control public expenditure and manage the government's cash balances. We will also introduce a new chart of accounts in the 2011 Budget with the Fund's technical assistance. We have set up a monitoring committee to follow up on the recommendations arising from audits conducted by the Auditor General (statutory) and internal auditors (ad hoc) in each government department on internal controls and procedures.

\section{Our fiscal policy objectives will be supported by strengthened budget processes.}

Starting with the 2011 Budget, we will move to a medium-term budgeting framework, presenting the government's fiscal and economic projections over a three-year horizon. The 2011 budget process will commence in June 2010 with Cabinet's discussion of a budget strategy document, setting the fiscal context and establishing the government's priorities. Ministries and other budget dependent entities will then submit their recurrent and capital expenditure proposals to the Ministry of Finance using a standard template that will detail the costing. The Ministry of Finance's capacity to scrutinize these expenditure proposals will also be enhanced through increased resources, the introduction of formal expenditure 
review procedures and detailed reconciliation of budget estimates with quarterly and yearend outcomes. In parallel, our macroeconomic and revenue forecasting capacity has been enhanced through the establishment of a dedicated branch within the Ministry of Finance which is now fully operational.

\section{We are also working towards a more complete presentation of the budget.}

The 2010 Budget document presented to National Assembly was more detailed than in previous years, including for the first time a full presentation of revenues, expenditure and financing, an annual borrowing plan and a medium-term debt strategy. The 2011 Budget will build on these achievements by presenting a more comprehensive analysis of the fiscal position and macroeconomic context and incorporating the Pension Fund into the general government accounts. A related work stream is the review of the operations of all public sector agencies, based on a classification by type of economic activity. Based on this classification, we have started to compile and publish consolidated general government accounts, consistent with international standards. It is also anticipated that this analysis will lead to the commercialization of some existing public sector agencies, in line with our broader strategy of reducing the role of the state in the economy.

\section{We are committed to improving public infrastructure, including addressing} urgent needs in the utilities sector. We will develop a memorandum of understanding between the government and Public Utilities Company (PUC) to promote strengthened collaboration on investment plans and tariff schedules in the utilities sector and will enhance financial oversight of PUC through the Public Enterprise Monitoring Division (PEMD) in the Ministry of Finance. While further work is required to identify priority investments in the utilities sector (e.g., electricity, water and sewage), following several years of underinvestment, the government's preliminary estimate is an investment need of over SR 2 billion over the next three years. The Public Utilities Company (PUC) has limited capacity to finance this investment internally, with the existing tariff structure - specifically, a substantial subsidy for household electricity consumption - insufficient to meet the costs of supply. The government's objective is to improve the quality of service provision in the utilities sector, which over the medium-term will require PUC to implement a tariff policy that is consistent with full cost recovery and meeting future investment needs. To this effect, we will commission a comprehensive study of the tariff structure, production costs and the quality of service provision in the utilities sector that will make recommendations to government on the optimal tariff policy in July 2011. The study will also include a social impact statement to inform the government's response. Meanwhile, most of the capital expenditure contingency is being directed to urgent utilities investment in 2010.

20. Our social safety net is successfully delivering targeted income support without undermining incentives to work. The number of income support recipients has decreased over the last year, in line with strengthened compliance and is expected to decline further as the economy recovers and job prospects improve. We have expanded training programs for the unemployed and those exiting the public service through the voluntary departure scheme 
to ensure that any period of unemployment is transitory. We are also working with the World Bank to fine-tune the targeting of our social safety net, including establishing a relationship to the minimum wage that preserves work incentives over time.

21. We will strengthen the Ministry of Finance's budgetary oversight of the Social Security Fund (SSF), improve the management of its assets, and initiate work on a medium-term strategy that will put our social security institutions (including the Pension Fund) on an actuarially sound basis. Today, the SSF has substantial financial assets, including sizeable cash balances with commercial banks. The financial operations of the SSF will change when the current social security contribution is replaced with the personal income tax. The Ministry of Finance will conduct a financial audit of the SSF, strengthen budgetary oversight of the SSF's financial operations and will help the SSF to rationalize its cash management. Concurrently, the World Bank is providing technical assistance on the institutional arrangements for merging the Social Security Fund and the Social Welfare Agency, reviewing the functions of the SSF and advising on the transfer of its assets and liabilities. Furthermore, as part of our medium-term reform program, we will also initiate an actuarial audit of the SSF as well as work on a comprehensive asset management strategy for the general government sector that aligns with the government's debt management strategy and takes into account accruing liabilities.

\section{Efforts to improve transparency and strengthen institutional capacity in the} area of fishing licenses remain a work in progress. There has been a marked decline in the number of licensed fishing vessels operating in our waters since 2008, when piracy in the Indian Ocean became more prevalent. Work is continuing on the disclosure of fishing license agreements and the construction of an economic and financial model to support more informed fishing licensing and natural resource management policies (see $q 27$ of previous MEFP).

\section{A. Monetary and Exchange Rate Policies}

23. Monetary policy will continue to be based on reserve money targeting through the use of indirect instruments. We will continue to use repos to guide short-term interest rates. Furthermore, we will be lengthening the maturity structure of the Deposit Auction Arrangement (DAA), which will provide for more flexibility in open market operations.

24. We are continuing to enhance coordination between monetary and fiscal policy to promote sound liquidity management and financial sector deepening. To this end, we will continue to make extensive use of the liquidity forecasting and reserve money programming framework. The CBS and the Ministry of Finance will continue to work closely on the T-bill issuance profile to promote the smooth functioning of the market and provide for the development of a yield curve. We will also communicate the calendar of Tbill issuance weekly for the following four week period and inform the market of the quantity of paper to be issued three days ahead of the auction to improve predictability and 
assist banks in better managing their liquidity. The CBS will also formalize the arrangements for its use of government securities as a monetary policy instrument through a memorandum of understanding with the Ministry of Finance by end-June 2010.

25. We remain committed to a floating exchange rate regime, which has served Seychelles well during the initial phases of our economic reforms. The CBS will maintain its policy of intervening in the foreign exchange market only to smooth out excessive volatility and ensure orderly market conditions. The CBS will continue to work closely with banks and make use of technical assistance from the IMF, to improve the efficiency of the inter-bank foreign exchange market. Within this context, we are working on a code of conduct which will guide all participants in the foreign exchange as well as in the money market.

\section{B. CBS Governance and Capitalization}

26. The implementation of an integrated (core) banking system is on course for completion by the fourth quarter of 2010. This project will assist the CBS to (i) significantly improve the efficiency of its operations; (ii) modernize banking services; and (iii) significantly minimize IT risks. The implementation and license agreements have been signed by CBS and the selected vendor and a detailed project plan has been agreed to by the two parties. Work has already started and a complete gap analysis is being conducted by the selected vendor with the assistance of the external auditors.

\section{The CBS is undercapitalized, which if left unaddressed, could constrain its} ability to implement prudent monetary policies. Significant valuation losses in 2009, due to the appreciation of the rupee, have affected CBS's distributable profits (R40.0 million). With the 2009 results, 50 percent of the distributable profit equivalent to R20.0 million was retained to build up CBS capital which currently stands at 2.68 percent of monetary liabilities. As per the CBS Act, the government will implement a one-time recapitalization in 2010, boosting CBS capitalization to 10 percent of monetary liabilities by the end of the year. This will strengthen the balance sheet of the CBS and enhance its portfolio of tradable securities to be used in open market operations.

28. As external reserves continue to accumulate, the CBS will need to continue to strengthen its reserve management activities. Given its internal capacity constraints, we consider it premature at this stage to use commercial asset managers to manage a portion of our reserves, and instead will use the services of the World Bank's Reserve Advisory and Management Program (RAMP) and the Asset Management services of the Bank for International Settlement (BIS). The World Bank and the BIS have been approached to conduct a peer review of the CBS' reserve management activities (investment guidelines, investment committee etc). These institutions are expected to provide technical assistance in the area of capacity building, training of CBS internal managers in order to adequately manage the Bank's reserves, as well as investing a portion of CBS' external reserves. 
29. A reputable international audit firm is conducting a thorough risk assessment of the central bank to improve its internal controls. As part of the assessment the team will also provide training to CBS officers in the way forward to address these risks. CBS management is committed to monitor risks on a permanent basis and to ensure that high risk areas are properly mitigated, and that the internal audit functions of the Bank are strengthened.

30. CBS plans to complete the introduction of a business continuity plan (BCP) by end-2010. A contract will be tendered by mid-June to a consulting firm to assist it in this exercise. The first BCP will cover the priority services offered by the CBS and this will later be expanded to incorporate all other services.

\section{Financial Sector Reforms}

31. We will continue our efforts towards implementing a risk-based approach to supervision, in line with the Fund technical assistance recommendations. This is expected to be fully operational by mid 2011. Frequent onsite examinations are being conducted as per the manuals developed with the IMF's technical assistance. Minimum capital for banks has been doubled, with a three year transition period granted to existing banks.

32. We remain committed to adopt the best supervisory practices in the region. CBS will implement the Banking Supervision Application software ${ }^{2}$ to strengthen its supervisory process, especially offsite supervision. This is expected to be fully operational beginning 2011.

33. The CBS continues to be proactive in its effort to safeguard the financial sector stability. At the request of CBS, the Toronto Centre ${ }^{3}$ has agreed to provide training on crisis preparedness, tentatively in August when the main sections of the crisis preparedness plan will be drafted. This will document the process to manage financial crises and promote coordination between authorities.

34. Seychelles will continue its efforts to promote transparency in the offshore financial sector through strengthened supervision by the central bank in coordination with the Seychelles International Business Authority, and the Financial Intelligence Unit. To that effect a two phase review is being conducted by the Global Forum on Transparency and Exchange of Information for Tax Purposes which is expected to be completed by September 2010.

\footnotetext{
${ }^{2}$ Developed initially by ESAF/SADC, which is continuously being upgraded.

${ }^{3}$ This is an institution that provides technical assistance in the area of financial supervision. Both the IMF and the World Bank are represented at the Board of the Toronto Centre.
} 
35. We are committed to encouraging a sound and efficient insurance sector that promotes policyholder confidence. The CBS has submitted to First Initiative a project proposal for technical assistance to strengthen the supervisory process of insurance supervision. This will include review of the regulatory framework and preparation of manuals for conducting examination of insurance and insurance-related companies.

36. We will continue to modernize the national payment system. Several important steps were taken in 2009 and in the first quarter of 2010, including the establishment of a complete requirements and standards document for electronic funds transfers that replaced the manual settlement system. The adoption of the new cheque standard documents in July 2009; the new clearing house rules in April 2010; and the introduction of the National Clearing and Settlement system bill in June 2010 will facilitate the introduction of the Electronic clearing house system. The CBS will continue to promote the use of electronic funds transfer and the use of plastic card, as well as implement a real time gross settlement system in 2011/2012.

\section{External Sector Policies}

\section{EXTERNAL DEBT RESTRUCTURING}

37. We are making considerable progress in normalizing relations with external creditors. To date, restructuring agreements covering approximately US\$575 million, or around $85 \%$ of the debt eligible for restructuring have been reached. In aggregate, these agreements have cancelled approximately US\$310 million in principal, accrued interest, and other charges. Remaining balances have by and large been rescheduled over periods exceeding 17 years at low rates of interest.

38. In February 2010, the country's commercial debt exchange offer closed successfully with a $50 \%$ debt cancellation. The transaction, which was launched in December 2009 following a period of discussions with key affected creditors, sought to restructure the bulk of Seychelles's external commercial debt. Claims with a face value of US\$320 million were eligible to participate in the exchange offer, including the US\$230 million Eurobond, the $€ 55$ million Amortizing Notes and two commercial bank loans held by three banks. The debt exchange was supported by a guarantee from the African Development Bank. Upon the expiry of the exchange offer period, $84 \%$ of the face value of the Eurobond had been tendered, along with $100 \%$ of the Amortizing Notes and the two bank loans. After the triggering of the collective action clause in the Eurobond by bondholders, Seychelles achieved 100\% participation for the exchange. This transaction alone cancelled approximately US\$225 million in principal, accrued interest, and other charges.

39. Seychelles will persevere with its efforts to conclude restructuring agreements on comparable terms with the few remaining commercial bank and non-Paris Club official bilateral creditors holding claims. Since the approval of the Extended Fund 
Facility, bilateral restructuring agreements have been reached with four commercial banks covering eight facilities. The three smallest loans in this category were bought back by Seychelles at steep discounts to face value, with the remainder being rescheduled at par over the long term at low interest rates. The vast majority of these claims were converted into new rupee-denominated loans or bonds. The Government has reached comprehensive restructuring agreements with Libya and Malaysia, and is close to reaching agreements with two other non-Paris Club official bilateral creditors. The Government expects to sign its remaining Paris Club bilateral agreements ahead of the second tranche of Paris Club debt cancellation on 1 July 2010.

\section{TRADE POLICIES}

40. We remain committed to further liberalize our foreign trade policies. We remain on track for the implementation of the COMESA common external tariff by 2013. As a preliminary step, we have introduced in the 2010 budget a common excise tax on petroleum, motor vehicles, alcohol and cigarettes that applies equally to imports and locally-produced items. A roadmap has been established and a chief negotiator has been appointed to prepare for the negotiations to accede to the World Trade Organization.

\section{E. Public Enterprise Reforms}

41. The government is pursuing a public enterprise reform strategy aimed at achieving greater efficiency, transparency and accountability. The Cabinet has approved a detailed work plan for the Public Enterprise Monitoring Division (PEMD) directed at reinforcing the monitoring and control of public enterprises, strengthening governance, reducing dependence on public funding and minimizing financial risks to the state. The legal basis for this work was established with the approval of the Public Enterprise Monitoring and Control Act (the Act) by the National Assembly in late 2009, mandating strengthened oversight and reporting arrangements for public enterprises and setting uniform governance rules. Financial audits will continue to be regularly examined by the Public Accounts Committee of the National Assembly, in line with the Act. The Public Enterprise Monitoring Division has finalized strategic assessments for the largest public entitiesdrawing on recently concluded external financial audits and management audits. All these public entities have submitted statements of intent and action plans. They have taken the necessary steps to establish the following committees: procurement and investment, audit and finance, and remuneration, in line with audit recommendations. We have also commissioned an international aviation consultant to conduct a strategic assessment of the future of Air Seychelles and its ancillary services. The consultant is scheduled to report findings to the government in August 2010.

42. The government, together with the CBS, is strengthening the management and oversight of public financial institutions and assessing the case for continued state ownership. It is government's policy to privatize Nouvobanq in the context of our medium- 
term strategy. Regulatory oversight of the Development Bank of Seychelles (DBS) and the Housing Finance Company (HFC) has shifted from the Ministry of Finance to the CBS, leveraging off the CBS' financial sector expertise. The First Initiative is scheduled to assess the government's strategic options with regard to both DBS and HFC in the second half of 2010. We recognize that, in the longer term, privatization of the two publically-owned deposit taking institutions - Seychelles Savings Bank and Nouvobanq - could bring new technology, strengthen management and fresh capital to our banking system, stimulate competition and enhance the quality of bank services. In the near-term, however, both institutions are performing well financially, present limited risk to the government's balance sheet and are playing a leading role in promoting financial intermediation and credit creation. An IFC mission is scheduled for August to explore the government's options with regard to Nouvobanq and add more definition to the time of its future sale.

\section{F. National Statistics}

43. We remain strongly committed to improving the quality, periodicity, and timeliness of macroeconomic and financial statistics. A new National Statistics Act (NSA) which will be presented to the Cabinet in June 2010, reinforcing the National Statistics Bureau's (NSB) independence and strengthening its mandate to collect data. We are moving to address deficiencies in key macroeconomic data - especially GDP estimates, and external sector statistics - to improve their usefulness for economic analysis and policy formulation. To this effect, a strategic plan will be adopted by end-January 2011.

We will bring Seychelles in line with international best practice, by aiming to implement the IMF's Special Data Dissemination Standard (SDDS) and the Balance of Payments and International Investment Position Manual 6 by 2011. The Ministry of Employment will reinforce its efforts to produce high-frequency labor market data. We have requested technical assistance from the IMF Statistics Department (on fiscal and national accounts) and the World Bank (to strengthen the management and operations of the NSB).

\section{G. Program Monitoring}

\section{The EFF-supported program will be monitored by semi-annual program} reviews, with quarterly quantitative performance criteria and disbursements. The quarterly quantitative performance criteria (QPCs) for 2010 are shown in Table 1 . The structural benchmarks for 2010 are shown in Table 2 . The nonzero ceilings on the contracting or guaranteeing of external debt are to allow for budgeted public project finance and program support from multilateral institutions exclusively.

45. The completion of the second review under the EFF will be based on meeting the QPCs at end-September 2010. The second program review will be based on meeting the end-September 2010 QPCs and is scheduled to be completed by end-December 2010. It will focus on the 2011 Budget, progress in implementing the tax reform, and in strengthening 
public financial management. Quarterly financing assurance reviews will continue as long as public debt arrears remain outstanding.

46. The attached Technical Memorandum of Understanding (TMU) defines the quantitative performance criteria, indicative targets and adjusters under the program. Seychelles will avoid introducing new exchange restrictions, multiple currency practices, or bilateral payments agreements in contradiction with Article VIII of the IMF's Articles of Agreement and imposing any import restrictions for balance of payments reasons. We stand ready to adopt any additional measures, in consultation with IMF staff, which may become necessary to ensure program success. 
Table 1. Seychelles: Quantitative Performance Criteria Under the EFF, December 2009-December 2010

(Millions of Seychelles rupees; end-of-period)

\begin{tabular}{|c|c|c|c|c|c|c|c|c|c|c|c|c|}
\hline & \multirow{2}{*}{\multicolumn{3}{|c|}{2009}} & \multicolumn{9}{|c|}{2010} \\
\hline & & & & \multicolumn{3}{|c|}{ March } & \multicolumn{2}{|c|}{ June } & \multicolumn{2}{|c|}{ September } & \multicolumn{2}{|c|}{ December } \\
\hline & Program & Adjusted & Actual & Program & Adjusted & Prel. & Program & Rev.prog. & Program & Rev.prog. & Program & Rev.prog. \\
\hline \multicolumn{13}{|l|}{ Performance criteria } \\
\hline Net international reserves of the CBS, millions of U.S. dollars (floor) ${ }^{1}$ & 130 & 138 & 153 & 133 & 137 & 192 & 128 & 128 & 142 & 142 & 168 & 168 \\
\hline Reserve money (ceiling) & 1,480 & $\ldots$ & 1,296 & 1,537 & $\ldots$ & 1,424 & 1,592 & 1,592 & 1,676 & 1,676 & 1,753 & 1,753 \\
\hline Primary balance of the consolidated government (cumulative floor) ( $^{2,3}$ & 1,375 & $\ldots$ & 1,586 & 261 & $\ldots$ & 298 & 437 & 455 & 659 & 668 & 761 & 823 \\
\hline $\begin{array}{l}\text { The contracting or guaranteeing of new external debt by the public sector } \\
\text { (Millions of U.S. dollars; cumulative ceiling) }{ }^{2}\end{array}$ & 39 & $\ldots$ & 30 & 2 & $\ldots$ & 0 & 3 & 3 & 13 & 13 & 37 & 37 \\
\hline $\begin{array}{l}\text { The contracting or guaranteeing of new short-term external debt by the public s } \\
\text { (Millions of U.S. dollars; cumulative ceiling) }{ }^{2}\end{array}$ & 0.0 & $\ldots$ & 0.0 & 0.0 & $\ldots$ & 0.0 & 0.0 & 0.0 & 0.0 & 0.0 & 0.0 & 0.0 \\
\hline The accumulation of external payments arrears by the public sector (ceiling) ${ }^{3}$ & 0.0 & $\ldots$ & 0.0 & 0.0 & $\ldots$ & 0.0 & 0.0 & 0.0 & 0.0 & 0.0 & 0.0 & 0.0 \\
\hline The accumulation of domestic payment arrears by the public sector (ceiling) & 0.0 & $\ldots$ & 0.0 & 0.0 & $\ldots$ & 0.0 & 0.0 & 0.0 & 0.0 & 0.0 & 0.0 & 0.0 \\
\hline \multicolumn{13}{|l|}{ Memorandum items: } \\
\hline External non project financing (millions of U.S. dollars; cumulative) ${ }^{2}$ & -17.2 & $\ldots$ & -9.3 & 1.7 & $\ldots$ & 5.6 & 1.8 & 1.8 & 9.0 & 9.0 & 31.8 & 31.8 \\
\hline Program financing support & 19.6 & $\ldots$ & 11.2 & 0.0 & $\ldots$ & 8.7 & 0.0 & 0.0 & 0.0 & 0.0 & 19.6 & 19.6 \\
\hline Cash payments on foreign debt service & 48.1 & $\ldots$ & 32.0 & 1.8 & $\ldots$ & 3.9 & 5.8 & 5.8 & 6.9 & 6.9 & 9.2 & 9.2 \\
\hline External budget grants & 11.3 & $\ldots$ & 11.5 & 3.5 & $\ldots$ & 0.8 & 7.6 & 7.6 & 15.9 & 15.9 & 21.4 & 21.4 \\
\hline \multicolumn{13}{|l|}{ Program accounting exchange rate } \\
\hline SR/US\$ (end-of-quarter) & 11.00 & $\ldots$ & 11.25 & 11.00 & $\ldots$ & 11.00 & 11.00 & 11.75 & 11.00 & 11.75 & 11.00 & 11.75 \\
\hline US\$/Euro (end-of-quarter) & 1.48 & $\ldots$ & 1.43 & 1.48 & $\ldots$ & 1.48 & 1.48 & 1.27 & 1.48 & 1.27 & 1.48 & 1.27 \\
\hline US $\$ /$ UK pound (end-of-quarter) & 1.65 & $\ldots$ & 1.62 & 1.65 & $\ldots$ & 1.65 & 1.65 & 1.48 & 1.65 & 1.48 & 1.65 & 1.48 \\
\hline US $\$ / S D R$ (end-of-quarter) & 1.59 & $\ldots$ & 1.57 & 1.59 & $\ldots$ & 1.59 & 1.59 & 1.49 & 1.59 & 1.49 & 1.59 & 1.49 \\
\hline
\end{tabular}

Sources: Seychelles authorities and IMF staff estimates and projections.

${ }^{1}$ The floor will be adjusted downward (upward) for any shortfall (excess) in external nonproject financial support from that assumed in the program.

${ }^{2}$ Cumulative flows from the beginning of the calendar year.

${ }^{3}$ The nonaccumulation of new external payment arrears constitutes a continuous performance criterion. Excludes arrears for which a rescheduling agreement is sought. 
Table 2. Seychelles: Structural Benchmarks, 2009-10

\begin{tabular}{|c|c|c|c|}
\hline Measure & Target date & Macroeconomic rationale & Status \\
\hline $\begin{array}{l}\text { Introduce a treasury single } \\
\text { account. }\end{array}$ & $\begin{array}{l}\text { End- } \\
\text { September } 2009\end{array}$ & $\begin{array}{l}\text { To strengthen public } \\
\text { finances, key to sustainability } \\
\text { efforts. }\end{array}$ & $\begin{array}{l}\text { Met. The TSA is } \\
\text { leveraging progress on } \\
\text { PFM. }\end{array}$ \\
\hline $\begin{array}{l}\text { Adopt foreign reserves } \\
\text { management investment } \\
\text { guidelines. }\end{array}$ & $\begin{array}{l}\text { End- } \\
\text { September } 2009\end{array}$ & $\begin{array}{l}\text { To ensure management of } \\
\text { foreign reserves in line with } \\
\text { best international practice. }\end{array}$ & $\begin{array}{l}\text { Met. The CBS Board } \\
\text { adopted guidelines on } \\
\text { September 25, } 2009 .\end{array}$ \\
\hline $\begin{array}{l}\text { Adopt Public Enterprise } \\
\text { Monitoring and Control act. }\end{array}$ & $\begin{array}{l}\text { End- } \\
\text { September } 2009\end{array}$ & $\begin{array}{l}\text { To improve oversight over } \\
\text { public enterprises and reduce } \\
\text { quasi-fiscal risks. }\end{array}$ & $\begin{array}{l}\text { Met. Parliamentary } \\
\text { approval secured on } \\
\text { September } 28,2009 \text {. }\end{array}$ \\
\hline $\begin{array}{l}\text { Complete CBS procedures } \\
\text { manual (MEFP, } \llbracket 46) \text {. }\end{array}$ & $\begin{array}{l}\text { End- } \\
\text { December } 2009\end{array}$ & $\begin{array}{l}\text { To support improved } \\
\text { efficiency and transparency } \\
\text { in monetary policy. }\end{array}$ & $\begin{array}{l}\text { Met. The manual was } \\
\text { approved on } \\
\text { December } 29,2009 .\end{array}$ \\
\hline 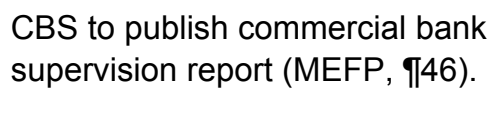 & $\begin{array}{l}\text { End- } \\
\text { December } 2009\end{array}$ & $\begin{array}{l}\text { To promote competition and } \\
\text { transparency in the banking } \\
\text { system. }\end{array}$ & $\begin{array}{l}\text { Met. Published on the } \\
\text { CBS website on } \\
\text { December 29, } 2009\end{array}$ \\
\hline $\begin{array}{l}\text { Amend the Business Tax Act in } \\
\text { line with the tax reform strategy } \\
\text { (MEFP, } \llbracket 22 \text { ). }\end{array}$ & $\begin{array}{l}\text { End- } \\
\text { December } 2009\end{array}$ & $\begin{array}{l}\text { To broaden the tax base, } \\
\text { modernize tax policy, and } \\
\text { remove distortions. }\end{array}$ & Met. \\
\hline $\begin{array}{l}\text { Cabinet approval of customs } \\
\text { reform strategy and } \\
\text { implementation plan (MEFP, } \\
\text { \23). }\end{array}$ & End-March 2010 & $\begin{array}{l}\text { To transform customs into a } \\
\text { modern and efficient entity. }\end{array}$ & $\begin{array}{l}\text { Met. The approval was } \\
\text { secured on } \\
\text { March 17, } 2010 .\end{array}$ \\
\hline $\begin{array}{l}\text { Publish general government } \\
\text { fiscal statistics (MEFP, } \mid 29 \text { ). }\end{array}$ & End-April 2010 & $\begin{array}{l}\text { To enhance transparency } \\
\text { and coverage of public } \\
\text { finance. }\end{array}$ & $\begin{array}{l}\text { Met. Fiscal statistics } \\
\text { were published on } \\
\text { April 23, } 2010\end{array}$ \\
\hline $\begin{array}{l}\text { Submit to National Assembly a } \\
\text { new customs management act } \\
\text { (MEFP, ๆ23). }\end{array}$ & End-June 2010 & $\begin{array}{l}\text { To institutionalize best } \\
\text { international practice and a } \\
\text { better business climate. }\end{array}$ & $\begin{array}{l}\text { Moved to end- } \\
\text { September 2010, to } \\
\text { address regional } \\
\text { harmonization issues. }\end{array}$ \\
\hline $\begin{array}{l}\text { Introduce Personal Income Tax } \\
\text { (MEFP, ף22). }\end{array}$ & July 1, 2010 & $\begin{array}{l}\text { To broader the tax base and } \\
\text { provide for more equitable } \\
\text { taxation. }\end{array}$ & \\
\hline $\begin{array}{l}\text { Introduce budget submissions } \\
\text { protocols and procedures } \\
\text { (MEFP, } \mid 28 \text { ). }\end{array}$ & End-July 2010 & $\begin{array}{l}\text { To strengthen budget } \\
\text { preparation. }\end{array}$ & \\
\hline 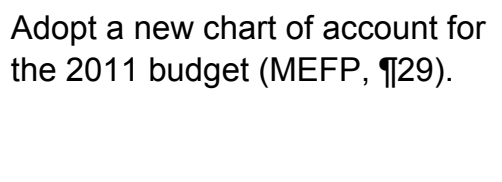 & $\begin{array}{l}\text { End- } \\
\text { November } 2010\end{array}$ & $\begin{array}{l}\text { To ensure proper } \\
\text { classification and increase } \\
\text { efficiency of the budget as a } \\
\text { policy tool. }\end{array}$ & \\
\hline $\begin{array}{l}\text { Submit to National Assembly a } \\
\text { bill creating a national clearing } \\
\text { house and settlement system } \\
\text { (MEFP, ๆ44). }\end{array}$ & $\begin{array}{l}\text { End- } \\
\text { December } 2010\end{array}$ & $\begin{array}{l}\text { To introduce necessary } \\
\text { financial infrastructure for } \\
\text { improved efficiency and } \\
\text { reduced risks. }\end{array}$ & \\
\hline
\end{tabular}


Table 3. Seychelles: Principal Structural Reform Measures Implemented in November 2009-April 2010

\section{Action}

Timing

Fiscal Policy

Increase in GST on tourism to 10 per cent

November 2009

Extend budget coverage for 2010 to include:

December 2009

full presentation of revenues, expenditure, and financing;

discussion of macroeconomic assumptions and objectives;

fiscal targets and objectives; and

an annual borrowing plan.

Publish a medium term debt strategy with the 2010 Budget

December 2009

Introduction of Revenue Administration Act and Business Number Act.

December 2009

Introduction of a revised Business Tax Act

December 2009

Reduction in cascading through levying GST on either imports or sales

December 2009

Partial conversion of Trade Taxes to Excise Duties (petroleum, cigarettes and alcohol)

January2010

Extend partial excise on petroleum to PUC

Introduction of a new public sector wage grid

Introduction of a new Budget calendar

Publication of monthly fiscal report on the Ministry of Finance website

Cabinet approval of the Customs Reform Strategy and implementation plan

January2010

January2010

March 2010

March 2010

March 2010

\section{Public Enterprise Reform}

Secure services of aviation consultant for strategic assessment of Air Seychelles

November 2009

Cabinet approval of a public enterprise reform strategy and work plan for PEMD

December 2009

Commence establishment of performance benchmarks for public enterprises

December 2009

\section{CBS Governance and Capitalization}

Completion of the CBS Procedures Manual

December 2009

Publication of the Commercial Bank Supervision Report

December 2009

Introduction of new methodology for calculating application and annual license fees for domestic and offshore banks

Adopt IFRS for 2009 CBS Accounts

March 2010

March 2010

\section{National Statistics}

Publication of the General Government Fiscal Statistics

April 2010 
Table 4. Seychelles: Principal Structural Reform Measures in Progress at May 2010

\section{Action}

Fiscal Policy

Cabinet approval of 2011 Budget Strategy

Introduction of a new Auditor General's Act

Establish guidelines for government guarantees

Introduce standardized tender bidding document for all public procurement

Half-yearly budget review of expenditure by all government departments

Establish a comprehensive Government Assets Register

Adopt a new chart of accounts for the 2011 Budget

Determine budget treatment of all budget dependent agencies

Budget management training for departmental budget officers

Revise Financial Instructions and Accounting Manual

Introduction of a new Public Finance Law

Review the Accounting Standards of Financial Statement to follow International

Public Sector Accounting Standards (IPSAS)

Build capacity in the Ministry of Finance to analyze capital expenditure proposals

\section{Tax Reforms}

Set up an audit group to focus on large taxpayers

Submit a new Customs Management Act to National Assembly

Increase GST on tourism to $12 \%$

\section{Public Enterprise Reform}

Commission external financial audit of DBS and HFC

Engage FIRST Initiative on strategic options assessment of DBS and HFC

Engagement of IFC to advise on the government's options on Nouvobanq

Implementation plan in response to external strategic assessment of Air Seychelles

Commission a comprehensive study of the tariff structure, production costs, and quality of service provision in the utilities sector

Implement completed reform action plans for major public enterprises

\section{CBS Governance and Capitalization}

Present Electronic Clearing and Settlement System Bill to National Assembly

Produce financial survey encompassing bank and nonbank financial institutions Introduce integrated IT-based accounting framework at CBS (core banking system)

Introduce a business continuity plan at CBS

Adopt a crisis preparedness plan for financial system

Progressively raise capitalization of CBS to 10 percent of monetary liabilities

Gradually raise minimum capital requirements for domestic banks.
Timing

June 2010

June 2010

June 2010

June 2010

July 2010

August 2010

November 2010

December 2010

Jan - Dec 2010

March 2011

September 2011

September 2011

2010-11

July 2010

September 2010

November 2010

June 2010

July 2010

August 2010

October 2010

2010

2010-11

June 2010

December 2010

December 2010

December 2010

December 2010

December 2010

2010-12 
Implementation of real time gross settlement system.

$2011-12$

National Statistics

Adopt a strategic plan to upgrade national statistics

September 2010

Implement Balance of Payments and International Investment Position Manual 6

2011

Implement SDDS

2011 


\section{AtTaChment II}

\section{SEYCHELlES: TECHNICAL MEMORANDUM OF UNDERSTANDING}

1. This technical memorandum of understanding presents the definitions of variables included in the quantitative performance criteria and indicative targets set out in the memorandum of economic and financial policies (SMEFP), the key assumptions, and the reporting requirements of the Government and the Central Bank of Seychelles (CBS) needed to adequately monitor economic and financial developments. The quantitative performance criteria and indicative targets, and the benchmarks for 2010 are listed in Tables 1 and 2 of the SMEFP, respectively.

\section{Quantitative Performance Criteria}

\section{A. Net International Reserves of the CBS (Floor)}

\section{Definition}

2. Net international reserves (NIR) of the CBS are defined for program monitoring purposes as reserve assets of the CBS, minus reserve liabilities of the CBS (including liabilities to the IMF). Reserve assets of the CBS are claims on nonresidents that are readily available (i.e., liquid and marketable assets, free of any pledges or encumberments and excluding project balances and blocked or escrow accounts, and bank reserves in foreign currency maintained for the purpose of meeting the reserve requirements), controlled by the CBS, and held for the purpose of intervening in foreign exchange markets. They include holdings of SDRs, holdings of foreign exchange, demand and short-term deposits at foreign banks abroad, fixed-term deposits abroad that can be liquidated without penalty, and any holdings of investment-grade securities. Reserve liabilities of the CBS comprise liabilities to nonresidents contracted by the CBS, any net off-balance sheet position of the CBS (futures, forwards, swaps, or options) with either residents or nonresidents, including those to the IMF.

\section{Calculation method}

3. For program monitoring purposes, reserves assets and liabilities at each test date, must be converted into U.S. dollars using the end of period exchange rates assumed in the program.

\section{Adjusters}

4. The floor on the CBS's NIR will be adjusted upward (downward) by the amount by which the external nonproject loans and cash grants exceeds (falls short of) the amounts assumed in the program (SMEFP Table 1). The floors will also be adjusted upwards (downwards) by the amount that external debt service payments fall short (exceed) the amounts assumed in the program. 


\section{B. Reserve Money (Ceiling)}

\section{Definition}

5. Reserve money is equivalent to currency issued and deposits held by financial institutions at the central bank (bank reserves), including those denominated in foreign currencies. Evaluation of performance of reserve money with respect to the program ceiling will be done at the program accounting exchange rate.

\section{Primary Balance of the Consolidated Government (Cumulative Floor)}

6. The consolidated government primary balance from above the line on a commitment basis is defined as total consolidated government and social security fund revenues (excluding privatization and long-term lease income receipts) less all noninterest (primary) expenditures of the government and social security fund.

\section{Public External Debt (Ceiling)}

7. The ceiling applies to the contracting or guaranteeing of new external liabilities by the public sector (including the central government, the CBS, and all public agencies and parastatals for operations that are not directly linked to commercial activities). The ceiling does not apply to the use of Fund resources, operations related to external debt restructuring; normal import related credits; purchases of treasury securities by nonresidents; or borrowing by parastatals in the conduct of normal commercial operations. The non zero ceilings on the contracting or guaranteeing of external debt are to allow for normal public project finance and program support from multilateral institutions exclusively. Debt shall be valued in U.S. dollars at program exchange rates. A zero sub-ceiling on short-term external debt applies continuously to the contracting or guaranteeing of short-term external debt by the public sector, with an original maturity of up to and including one year.

8. For the purpose of this performance criterion, external debt is defined as foreign currency denominated debt, and the term "debt" has the meaning set forth in point No. 9 of the Executive Board's Guidelines on Performance Criteria with Respect to Foreign Debt (Decision No. 12274-(00/85)). Debt is understood to mean a current, non contingent liability, created under a contractual arrangement through the provision of value in the form of assets (including currency) or services, and which requires the obligor to make one or more payments in the form of assets (including currency) or services, at some future points in time; these payments will discharge the principal and/or interest liabilities incurred under the contract. The ceiling on contracting official and officially guaranteed external debt includes all form of debt, including:

(i) loans, i.e., advances of money to the obligor by the lender made on the basis of an undertaking that the obligor will repay the funds in the future (including deposits, bonds, debentures, commercial loans, and buyers credits) and temporary exchanges of 
assets that are equivalent to fully collateralized loans under which the obligor is required to repay the funds, and usually pay interest, by repurchasing the collateral from the buyer in the future (such as repurchase agreements and official swap arrangements);

(ii) suppliers credits, i.e., contracts where the supplier permits the obligor to defer payments until some time after the date on which the goods are delivered or services are provided;

(iii) leases, i.e., arrangements under which property is provided which the lessee has the right to use for one or more specified period(s) of time that are usually shorter than the total expected service life of the property, while the leasor retains title to the property. The debt is the present value (at the inception of the lease) of all lease payments expected to be made during the period of the agreement excluding those payments that cover the operation, repair, or maintenance of the property; and

(iv) Arrears, penalties, and judicially awarded damages arising from the failure to make payment under a contractual obligation that constitutes debt are debt.

\section{E. External Arrears of the Public Sector}

9. The nonaccumulation of arrears to external creditors will be a continuous performance criterion under the program. External payments arrears for program monitoring purposes are defined as the amount of external debt service due and not paid within the contractually agreed period, including contractual and late interest. Arrears resulting from the nonpayment of debt service for which a clearance framework has been agreed or a rescheduling agreement is sought are excluded from this definition.

\section{F. Budget Expenditure Arrears}

10. The nonaccumulation of budget expenditure arrears will be a continuous performance criterion under the program. Budget expenditure arrears are defined as the sum of (1) any invoice that has been received by a spending agency from a supplier of goods, services, and capital goods delivered and verified, and for which payment has not been made within the contractually agreed period, or in the absence of a grace period, within 30 days; (2) unpaid wages, pensions, or transfers, pending for longer than 30 days to domestic or foreign residents, irrespective of the currency denomination of the debt.

\section{DATA AND INFORMATION}

11. The Seychelles authorities (government and CBS) will provide Fund staff with the following data and information according to the schedule provided.

\section{The CBS will report}

Weekly (within one week from the end of the period) 
- $\quad$ Reserve money.

- Foreign exchange reserves position.

- A summary table on the foreign exchange market transactions.

- The results of the liquidity deposit auctions, primary treasury bill auctions, and secondary auctions.

Monthly (within four weeks from the end of the month)

- The monetary survey in the standardized report form format.

- The foreign exchange cash flow, actual and updated.

- Financial soundness indicators.

- Stock of government securities in circulation by holder (banks and nonbanks) and by original maturity and the debt service profile report.

\section{The Ministry of Finance will report}

Monthly (within two weeks from the end of the month):

- Consolidated government operations on a commitment basis and cash basis in the IMF-supported program format.

- The detailed revenues and expenditures of the central government and social security fund.

- Monthly accounts of the public nonbank financial institutions.

- Import and export data from the customs department.

- Monthly public debt report.

The government and CBS will consult with Fund staff on all economic and financial measures that would have an impact on program implementation, and will provide any additional relevant information as requested by Fund staff. 


\section{INTERNATIONAL MONETARY FUND}

\section{SEYCHELLES}

First Review Under the Extended Arrangement, Request for Modification of Performance Criteria, and Financing Assurances Review_-Informational Annex

Prepared by the African Department

(In consultation with other departments)

June 15,2010

Contents

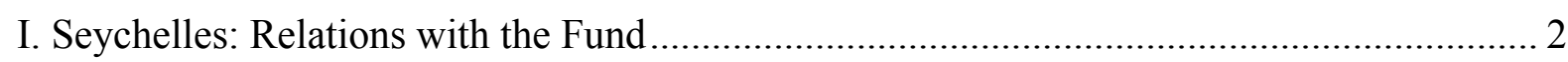

II. Seychelles: Joint World Bank-IMF Work Program, 2010 .................................................. 6

III. Seychelles: Relations with the African Development Bank Group .................................... 8 


\section{SEYCHELLES: RELATIONS WITH THE FUND}

(As of April 30, 2010)

I. Membership Status: Joined 6/30/77. Article VIII.

II. General Resources Account

Quota

Fund holdings of currency

Reserve Position in Fund

III. SDR Department

Net cumulative allocation

Holdings

\begin{tabular}{lll} 
SDR Million & & \% Quota \\
\hline 8.80 & & 100.0 \\
22.88 & & 259.97 \\
0.00 & & 0.04
\end{tabular}

$\underline{\text { SDR Million }}$

8.28

$\%$ Allocations

100.0

7.80

IV. Outstanding Purchases and

Loans:

Stand-by Arrangement

11.00

125.00

Extended Arrangement

3.08

35.00

V. Financial Arrangements:

$\underline{\text { SDR Million }}$

Type Arrangement Expiration Amount Approved Amount Drawn

$\begin{array}{lrrrr}\text { EFF } & \text { Dec 23, 2009 } & \text { Dec. 22, 2012 } & 19.80 & 3.08 \\ \text { Stand-By } & \text { Nov.14, 2008 } & \text { Dec. 22, 2009 } & 17.60 & 11.00\end{array}$

VI. Projected Obligations to the Fund (SDR Million: based on existing use of resources and present holdings of SDRs):

\begin{tabular}{l|ccccc}
\hline & \multicolumn{5}{|c}{ Forthcoming } \\
\hline & $\mathbf{2 0 1 0}$ & $\mathbf{2 0 1 1}$ & $\mathbf{2 0 1 2}$ & $\mathbf{2 0 1 3}$ & $\mathbf{2 0 1 4}$ \\
Principal & & & 3.41 & 5.50 & 2.42 \\
Charges/interest & 0.13 & 0.18 & 0.17 & 0.11 & 0.06 \\
Total & 0.13 & 0.18 & 3.58 & 5.61 & 2.48 \\
\hline
\end{tabular}


VII. Implementation of HIPC Initiative: Not applicable

\section{Safeguards Assessments:}

The Central Bank of Seychelles (CBS) was subject to a safeguards assessment with respect to the Stand-By Arrangement for Seychelles. The assessment completed in 2008 found high risks in all areas of the CBS's safeguards framework, and several measures were included in the program. An update assessment in relation to the Extended Fund Facility, which is underway, has found that the authorities have implemented most of the measures recommended by the 2008 assessment. In particular, governance oversight at the CBS is now exercised by the Board and management committees, and transparency improved through application of International Financial Reporting Standards and the publication of financial statements that have been audited in accordance with international standards. Some vulnerability remains, however, for which remedial measures are proposed.

\section{Exchange Rate Arrangement:}

The exchange market was liberalized in November 2008 and resulted in elimination of all the restrictions on the making of payments and transfers for current international transactions that are subject to Fund approval under Article VIII Sections 2, 3, and 4. The exchange rate policy is floating. On May 27, 2010, US\$1 = SR 12.33 (mid rate).

\section{Article IV Consultations:}

Seychelles is currently under a three year Extended Arrangement, with semi-annual reviews. 


\section{Technical Assistance (2004-March 2010):}

\begin{tabular}{|c|c|c|c|}
\hline Department & Head of Mission & Subject & Date \\
\hline STA & Mr. Freeman & National Accounts/CPI & May/June 2005 \\
\hline MFD/LEG & Mr. Lonnberg & $\begin{array}{l}\text { Strengthening Capacity in } \\
\text { Critical Central Banking } \\
\text { Areas, including } \\
\text { AML/CFT-Legal, } \\
\text { Institutional, and } \\
\text { Supervisory Frameworks }\end{array}$ & July 2006 \\
\hline LEG & Mr. Beekarry & $\begin{array}{l}\text { Advise on AML/CFT } \\
\text { measures and the } \\
\text { establishment of the FIU }\end{array}$ & $\begin{array}{l}\text { September/October } \\
2006\end{array}$ \\
\hline STA & Mr. Alexander & $\begin{array}{l}\text { National } \\
\text { accounts/CPI/GDDS }\end{array}$ & $\begin{array}{l}\text { October/ } \\
\text { November } 2006\end{array}$ \\
\hline MCM & Mr. Bartholomew & $\begin{array}{l}\text { Multipurpose mission: } \\
\text { Monetary operations, } \\
\text { monetary research, } \\
\text { banking supervision, } \\
\text { payment systems, foreign } \\
\text { exchange markets, and } \\
\text { nonbank financial } \\
\text { institution supervision. }\end{array}$ & $\begin{array}{l}\text { November/ } \\
\text { December } 2007\end{array}$ \\
\hline STA & Mr. Dessart & $\begin{array}{l}\text { Dissemination of GDDS } \\
\text { National Summary Data } \\
\text { Page }\end{array}$ & April 2008 \\
\hline STA & Mr. Armknecht & Consumer price index & April 2008 \\
\hline MCM & Mr. Faulk & $\begin{array}{l}\text { Banking supervision, } \\
\text { drafting of new financial } \\
\text { sector related regulations }\end{array}$ & April 2008 \\
\hline MCM & Mr. Robotham & $\begin{array}{l}\text { National payment systems } \\
\text { project }\end{array}$ & May/June 2008 \\
\hline FAD & Mr. Khemani & $\begin{array}{l}\text { Strengthening expenditure } \\
\text { rationalization and budget } \\
\text { management }\end{array}$ & July 2008 \\
\hline LEG & Mr. Baban & $\begin{array}{l}\text { Exchange Rate and } \\
\text { Exchange Control } \\
\text { Regimes }\end{array}$ & September 2008 \\
\hline
\end{tabular}




\begin{tabular}{|c|c|c|c|}
\hline Department & Head of Mission & Subject & Date \\
\hline FIN & Mr. Hauge & Safeguards assessment & October 2008 \\
\hline FAD & Mr. Krelove & Reform of Tax Policy & $\begin{array}{l}\text { January/February } \\
2009\end{array}$ \\
\hline MCM & Mr. Bartholomew & $\begin{array}{l}\text { Central Bank Capacity } \\
\text { Building after the } 2008 \\
\text { Float }\end{array}$ & February 2009 \\
\hline LEG & Mr. Baban & $\begin{array}{l}\text { Reform of Exchange Rate } \\
\text { Law }\end{array}$ & May 2009 \\
\hline STA & Mr. Jones & $\begin{array}{l}\text { Government Finance } \\
\text { Statistics }\end{array}$ & May 2009 \\
\hline MCM & Mr. Faulk & Bank Supervision & June 2009 \\
\hline $\mathrm{MCM}$ & Mr. Vollan & Monetary Operations & June 2009 \\
\hline $\mathrm{MCM}$ & Mr. Robotham & Payments Systems & June 2009 \\
\hline FAD & Mr. Ljungman & $\begin{array}{l}\text { Public Financial } \\
\text { Management }\end{array}$ & July 2009 \\
\hline LEG & Mr. Burns & Fiscal Law & July 2009 \\
\hline FAD & Mr. Kloeden & Tax Administration & September 2009 \\
\hline MCM & Mr. Sullivan & Accounting & October 2009 \\
\hline MCM & Mr. Faulk & Bank Supervision & October 2009 \\
\hline MCM & Mr. Robotham & Payments Systems & November 2009 \\
\hline STA & Ms. Razin & BoP Statistics & December 2009 \\
\hline MCM & Mr. Bartholomew & $\begin{array}{l}\text { Central Bank Capacity } \\
\text { Building }\end{array}$ & March 2010 \\
\hline
\end{tabular}

XII. Resident Representative None 


\section{Seychelles: JoInt World BANK-IMF WORK Program, 2010}

(As of May 31, 2010)

\begin{tabular}{|c|c|c|c|}
\hline Title & Products & $\begin{array}{l}\text { Provisional timing of } \\
\text { mission }\end{array}$ & $\begin{array}{l}\text { Expected delivery } \\
\text { date }\end{array}$ \\
\hline \multicolumn{4}{|c|}{ A. Mutual Information on Relevant Work Programs } \\
\hline Bank & $\begin{array}{l}\text { A. Strategy and analytical work } \\
\text { Public Expenditure Review (PER, Phase 2) } \\
\text { Joint Country Procurement Assessment Report } \\
\text { (CPAR) and Country Financial Accountability } \\
\text { Assessment (CFAA) } \\
\text { Tuna industry management (TA - P118247) } \\
\text { National Climate Change Strategy (TA - P118246) } \\
\text { B. Ongoing and new projects } \\
\text { GEF grant implementation (tsunami reconstruction, } \\
\text { disaster management) } \\
\text { Development Policy Loan (DPL 2) } \\
\text { Statistical Capacity Building Project (STATCAP) } \\
\text { Technical assistance on social security net and } \\
\text { enhancing targeting of social assistance }\end{array}$ & $\begin{array}{l}\text { July } 2010 \\
\text { n.a. } \\
\text { March } 2010 \\
\text { March } 2010 \\
\text { March } 2010 \\
\text { June } 2010\end{array}$ & $\begin{array}{l}\text { September } 2010 \\
\text { April } 2011 \\
\text { October } 2010 \\
\text { October } 2012 \\
\text { July } 2010\end{array}$ \\
\hline Fund & $\begin{array}{l}\text { Program and surveillance work } \\
\text { 1. First Review under EFF } \\
\text { 2. Article IV Consultation and Second Review under } \\
\text { EFF, including Debt Sustainability Analysis } \\
\text { Technical assistance and capacity building } \\
\text { 1. FAD technical assistance on tax and customs reform } \\
\text { 2. MCM technical assistance on reforming the } \\
\text { monetary policy framework (long-term monetary } \\
\text { advisor, automation of interbank market, reserve } \\
\text { money management) strengthening bank supervision, } \\
\text { payments systems, and CBS governance. }\end{array}$ & $\begin{array}{l}\text { May } 2010 \\
\text { October } 2010 \\
\text { Ongoing } \\
\text { Ongoing }\end{array}$ & $\begin{array}{l}\text { June } 2010 \\
\text { December } 2010 \\
\text { Ongoing } \\
\text { Ongoing }\end{array}$ \\
\hline Title & Products & $\begin{array}{l}\text { Provisional timing of } \\
\text { mission }\end{array}$ & $\begin{array}{l}\text { Expected delivery } \\
\text { date }\end{array}$ \\
\hline \multicolumn{4}{|c|}{ B. Requests for Work Program inputs } \\
\hline $\begin{array}{l}\text { Bank } \\
\text { request to } \\
\text { Fund }\end{array}$ & $\begin{array}{l}\text { 1. Medium-term macro-economic and fiscal framework } \\
\text { to inform Public Expenditure Reviews }\end{array}$ & n.a. & Ongoing \\
\hline
\end{tabular}




\begin{tabular}{|l|l|l|l|}
\hline $\begin{array}{l}\text { Fund } \\
\text { request to } \\
\text { Bank }\end{array}$ & $\begin{array}{l}\text { 1. IFC assistance on strategic plans for Nouvobanq } \\
\text { 2. FIRST Initiative support for strategic reform of } \\
\text { government owned non-bank financial institutions } \\
\text { 4. Strategy on improving business environment and } \\
\text { promoting private sector investment } \\
\text { 5. Develop strategy and policy recommendations to } \\
\text { improve transparency and management of the fisheries } \\
\text { sector }\end{array}$ & Ongoing & Jugust 2011 \\
\hline & $\begin{array}{l}\text { Joint mission in the context of the second review under } \\
\text { the EFF to discuss a medium-term structural reform } \\
\text { agenda focusing on: social safety net targeting; civil } \\
\text { service reform, parastatal reform; and promoting } \\
\text { private sector investment }\end{array}$ & 2010 \\
\hline $\begin{array}{l}\text { Joint } \\
\text { products }\end{array}$ & October 2010 & December 2010 \\
\hline
\end{tabular}




\section{SEyChelles: Relations With the African DeVelopMent Bank Group}

(As of May 31, 2010)

The African Development Bank Group commenced operations with the Republic of Seychelles in 1978 and has since approved 19 operations comprising 15 projects, one study and three lines of credit for the country. Cumulative approvals as of May 2010 (there were no approvals between 2001 and 2006 when the country was under sanctions) amounted to UA 84.87 million, ${ }^{1}$ of which 73 percent was from the African Development Bank (ADB), 14 percent from the Nigeria Trust Fund (NTF), and the remaining 13 percent from the African Development Fund (ADF) (Table 1). Reflecting government priorities, most of the operations were in the social sector (46 percent). This was followed by the financial sector (16 percent), agricultural sector (15 percent), transportation (13 percent), water supply and sanitation (10 percent) and industrial sector (1 percent). Before sanctions were imposed, about 76.8 percent of the total commitments on all approved loans had been disbursed and the balance of 23.2 percent was cancelled after sanctions were applied.

The country was under sanctions with the African Development Bank Group since February 16,2000 due to the arrears situation. The country cleared all the arrears (amounting to US\$46.75 million) in November 2006 and the African Development Bank Group consequently lifted all sanctions and started discussions on possible reengagement with the country. The process was boosted by the launching of a comprehensive reform program by the Government in November 2008. Following this, an Interim Strategy Note was approved by the Board on April 22, 2009. The ISN covers 2009 and 2010 and focuses on a single pillar namely supporting the Government's reform program to achieve sustainable growth. The lending program during this period consists of a policy-based operation in the form of a budget support loan amounting to $€ 15$ million, to be disbursed in two equal tranches of $€ 7.5$ million in 2009 and 2010. The operation was approved in July 2009 and the first tranche was disbursed in November 2009 while the second and final tranche is expected to be disbursed in the third quarter of 2010. In addition, there is an ongoing non-lending program under which the Bank Group is providing grant resources to the country, from the African Water Facility and the Middle Income Countries' (MIC) Technical Assistance Fund. Under the African Water Facility, a grant amounting to $€ 955,000$ was signed in May 2008 to finance the preparation of the Water Development Plan aimed at improved water management and governance, and identifying priority investment projects for funding, in order to meet Seychelles' water needs up to 2030. The study is expected to be completed by December 2010. Under the MIC Technical Assistance Fund two grants have been approved to finance:

\footnotetext{
${ }^{1} \mathrm{UA} 1=\mathrm{US} \$ 1.51112$ as at May $31,2010$.
} 
(i) a study (amounting to UA 600,000) approved in December 2008 aimed mainly to support human resource development, training and capacity building as well as the establishment of the Seychelles University Foundation; and (ii) a study (amounting to US\$ 500,000) approved in February 2009 to undertake the feasibility on a Submarine Cable System to link Seychelles to East Africa has already been completed. Another study on the Development of a Mariculture Master Plan (amounting to UA 280,000) is being processed.

The Bank approved an unprecedented policy based partial guarantee operation for US $\$ 10$ million on December 12, 2009 in support of an exchange bond offered to external commercial creditors in the context of the public debt restructuring effort. The guarantee was instrumental in generating a high participation in the debt exchange offer.

Table 1. Operations Summary as at 31 May 2010*

Operations since: 1978

Total Number of operations 19: 15 Projects, 1 Study; 3 Lines of Credit

\begin{tabular}{lrrrr}
\hline $\begin{array}{c}\text { Source of } \\
\text { Financing }\end{array}$ & \multicolumn{2}{c}{$\begin{array}{c}\text { Commitments } \\
\text { (UA million) }\end{array}$} & $\begin{array}{c}\text { Percentage } \\
\text { Share }\end{array}$ & $\begin{array}{c}\text { Disbursed } \\
\text { Amount } \\
\text { (UA million) Disbursed }\end{array}$ \\
& & & & \\
\hline ADB & 62.19 & 73 & 44.12 & 70.9 \\
ADF/TAF & 10.68 & 13 & 10.68 & 100.0 \\
NTF & 12.00 & 14 & 10.39 & 86.6 \\
Total & 84.87 & 100 & 65.19 & 76.8 \\
\hline
\end{tabular}

\footnotetext{
* Excludes the grants from the African Water Facility and the MIC Technical Assistance Facility due to their unique nature and small
} magnitude. 
Press Release No. 10/269

FOR IMMEDIATE RELEASE

June 30, 2010
International Monetary Fund

Washington, D.C. 20431 USA

\section{IMF Completes the First Review Under the Extended Fund Facility Arrangement for Seychelles and Approves US\$3.3 Million Disbursement}

The Executive Board of the International Monetary Fund (IMF) has completed the first review of the Seychelles' economic performance under the three-year Extended Fund Facility Arrangement (EFF). The completion of the review makes available a disbursement of SDR 2.2 million (about US\$3.3 million dollars), bringing total disbursements under the arrangement to SDR 5.28 million (about US\$7.8 million). The Board's decision was taken on a lapse of time basis. ${ }^{1}$ The EFF for Seychelles became effective on December 23, 2009 in the amount of SDR 19.8 million (about US\$29.3 million, or 225 percent of Seychelles' quota in the Fund (see Press Release No. 09/472).

The Seychelles economy has proved remarkably resilient to the double crisis it experienced. Following a turnaround in its economic policies to address an external debt crisis and a global financial crisis in 2008 , the country appears to have emerged from the recession. Real gross domestic product (GDP) grew by 0.7 percent in 2009 and growth is projected to recover further to 4 percent in 2010 .

The program remains fully on track and all quantitative performance criteria at end-March 2010 were met. Economic policies continue to be anchored by prudent fiscal policies. Government revenue is exceeding expectations in 2010, facilitating the implementation of key public infrastructure projects, while the decision to save part of the revenue overperformance will strengthen fiscal and external sustainability. The maintenance of prudent policies has given credibility to the currency float introduced in November 2008. Inflation has virtually disappeared, allowing a reduction in key policy interest rates, and international reserves are being rebuilt.

Progress on structural reforms is also good. On tax reforms, the business tax was rationalized and a personal income tax introduced. The next challenges will be to further broaden the tax base, reduce tax rates, modernize customs, and introduce a value-added tax in 2012. The

\footnotetext{
${ }^{1}$ The Executive Board takes decisions under its lapse of time procedure when it is agreed by the Board that a proposal can be considered without convening formal discussions.
}

Washington, D.C. 20431 • Telephone 202-623-7100 • Fax 202-623-6772 • www.imf.org 
authorities have also strengthened public finance and central bank management and are starting to address the restructuring of several key public enterprises and financial institutions, with a view to reducing the risks to the government budget and fostering sustained private sector-led growth.

Restructuring of Seychelles' external debt is progressing well. Strong policy implementation has convinced most external creditors to bring back external debt to sustainable levels, including through the completion of a debt exchange with private creditors in February 2010. 